\title{
An Enhanced Algorithm to Solve Multiserver Retrial Queueing Systems with Impatient Customers
}

\author{
Tien Van Do ${ }^{a, *}$ Nam H. Do ${ }^{b}$ Jie Zhang ${ }^{\mathrm{c}}$ \\ a MTA-BME Information Systems Research Group, \\ Department of Networked Systems and Services, \\ Budapest University of Technology and Economics, \\ H-1117, Magyar tudósok körútja 2., Budapest, Hungary. \\ ${ }^{\mathrm{b}}$ Inter-University Centre for Telecommunications and Informatics, Budapest \\ University of Technology and Economics, 4028 Debrecen, Kassai út 26., Hungary \\ ${ }^{\mathrm{c}}$ Communications Group, the Department of Electronic and Electrical Engineering, \\ University of Sheffield, Mappin Street, Sheffield, S1 3JD UK
}

\begin{abstract}
The homogenization of the state space for solving retrial queues refers to an approach where the performance of the $\mathrm{M} / \mathrm{M} / \mathrm{c}$ retrial queue with impatient customers and $c$ servers is approximated with a retrial queue with a maximum retrial rate restricted beyond a given number of users in the orbit. As a consequence, the stationary distribution can be obtained by the matrix-geometric method, which requires the computation of the rate matrix. In this paper, we revisit an approach based on the homogenization of the state space. We provide the exact expression for the conditional mean number of customers based on the computation of the rate matrix $R$ with the time complexity of $O(c)$. We develop simplified equations for the memory-efficient implementation of the computation of the performance measures. We construct an efficient algorithm for the stationary distribution with the determination of a threshold that allows the computation of performance measures with a specific accuracy.
\end{abstract}

Keywords: retrial queues, matrix-geometric method, spectral expansion, efficient algorithm

T. V. Do, N. H. Do, J. Zhang. An Enhanced Algorithm to Solve Multiserver Retrial Queueing Systems with Impatient Customers. Computers $8 \mathcal{G}$ Industrial Engineering, DOI:10.1016/j.cie.2013.04.008, 2013

* Corresponding author
Email addresses: do@hit.bme.hu (Tien Van Do), dohoai@hit.bme.hu (Nam H.

Accepted to Computers \& Industrial Engineering 


\section{Introduction}

Retrial queues have been used to take into account a phenomenon in modern information and telecommunication systems that blocked customers may rerequest for service after a certain timeout [1-10]. In retrial queues a client who does not receive the allocation of a server joins the orbit and later initiates a request for service. The $\mathrm{M} / \mathrm{M} / \mathrm{c}$ retrial queue has been analyzed by many researchers because the stationary distribution when the number of servers is larger than two can be only obtained using approximate techniques $[1,6-$ $8,11,12]$.

Falin [13] presented necessary and sufficient conditions for ergodicity of the retrial queues $M / M / c$. A well-known approximation is based on the truncation of the state space at a sufficiently large level related to the number of customers in the orbit [13]. Another approximation based on the homogenization of the model was pioneered by Neuts and Rao [14], where the $M / M / c$ retrial queue is approximated by the multiserver retrial queue with the total retrial rate that does not depend on the number of clients in the orbit as long as the orbit contains the number of clients greater than the specified value $N$. Note that the discussion for the choice of $N$ is presented in the recent book by Artalejo and Gómez-Corral on retrial queues [8]. With this assumption, the stationary probabilities of the $\mathrm{M} / \mathrm{M} / \mathrm{c}$ retrial queue can be estimated by any algorithm [15-19] based on the matrix-geometric method (MGM).

Recently, Domenech-Benlloch et al. [20] considered a multiserver retrial queue with the impatient phenomenon of customers waiting in the orbit. They proposed two different generalized truncated methods (called HM1 and HM2) based on the homogenization of the state space beyond a given number of users in the retrial orbit. The steady-state probabilities of the multiserver retrial queue with impatient customers are approximated with a modified retrial queue where the retrial rate beyond a certain level only depends on the conditional mean value of the number of customers in the orbit. Domenech-Benlloch et al. [20] also compared their methods with other well-known algorithms that belong to different categories [11] (approximations, finite truncated methods, generalized truncated methods). The authors [20] showed that the proposed HM2 method outperforms previous approaches from the aspect of accuracy at the price of increasing computation cost.

Based on the HM2 algorithm of Domenech-Benlloch et al. [20], our contributions allow an efficient computation for the stationary distribution and the performance measures. First, we revisit an approach based on the homogenization of the state space and provide an efficient method with the time complexity of only $O(c)$ to compute the rate matrix $R$. The method is based

Do). 
on a property that the characteristic matrix polynomial has only a single nonzero eigenvalue and this single non-zero eigenvalue can be computed using the bisection method. Second, we derive an exact expression for the conditional mean number of customers. Third, we develop simplified equations that allow the memory-efficient implementation of the computation of the performance measures. Fourth, we construct an efficient computation for the stationary distribution with the determination of a threshold, which guarantees a specific accuracy for the computation of performance measures.

The rest of this paper is organized as follows. In Section 2 we summarize the considered queueing model with impatient customers. In Section 3 we present our new results that serve as the foundations of the computation. In Section 4 we provide some numerical results to illustrate the efficiency of our algorithm. Finally, Section 5 concludes our paper.

\section{A Retrial Queueing Model with Impatient Customers}

We consider a retrial queueing model with $c$ homogenous servers and impatient customers. Inter-arrival times of customers are exponentially distributed with parameter $\lambda$. Holding times are exponentially distributed with parameter $\mu$. Random variable $I(t)$ represents the number of occupied servers at time $t$, hence $0 \leq I(t) \leq c$ holds. A client joins the orbit in order to wait and retry upon when $\Xi(t)=c$. Let $\neg(t)$ be the number of clients in the orbit waiting for retrial at time $t$. Each customer retries with rate $\mu_{r}$. Hence, the total effective retrial rate, when $\neg(t)=j$, is $j \mu_{r}$. A retrying customer either leaves the queue with probability $P_{i m}$ if all servers are busy upon the retrial or rejoins the orbit with probability $1-P_{i m}$. Note that a time between subsequent retrials of a specific user follows the exponential distribution with parameter $\mu_{r}$.

This system can be represented by two-dimensional continuous-time Markov chain $(\mathrm{CTMC}) Y=\{\beth(t)\urcorner,(t)\}$ with state space $\{0,1, \ldots, c\} \times\{0,1, \ldots\}$. Let the steady-state probabilities of CTMC $Y$ be denoted by $\pi_{i, j}=$ $\left.\lim _{t \rightarrow \infty} \operatorname{Pr}(\beth(t)=i\urcorner,(t)=j\right)$. Define the row vector $\mathbf{v}_{j}=\left[\pi_{0, j}, \ldots, \pi_{c, j}\right]$.

\subsection{Notations}

CTMC $Y$ is driven by the following transitions.

(a) $A_{j}(i, k)$ denotes the transition rate from state $(i, j)$ to state $(k, j)(0 \leq$ $i, k \leq c ; j=0,1, \ldots)$, which is caused by either the arrival of a customer (when $i<c$ ) or the leaving of a client after the expiry of a holding time. 
Matrix $A_{j}$ is of size $(c+1) \times(c+1)$ with elements $A_{j}(i, k)$. Since $A_{j}$ is $j$-independent, it can be written as $A_{j}=A$. The nonzero elements of $A_{j}$ are $A_{j}(i, i-1)=i \mu$ for $i=1, \ldots, c+1$, and $A_{j}(i, i+1)=\lambda$ for $i=0, \ldots, c$. Because $A_{j}$ is $j$-independent, it can be written as

$$
A_{j}=A=\left[\begin{array}{lllllll}
0 & \lambda & 0 & \ldots & 0 & 0 & 0 \\
\mu & 0 & \lambda & \ldots & 0 & 0 & 0 \\
\vdots & \vdots & \vdots & \vdots & \vdots & \vdots & \vdots \\
0 & 0 & \ldots & (c-1) \mu & 0 & \lambda \\
0 & 0 & \ldots & 0 & c \mu & 0
\end{array}\right], \forall j \geq 0
$$

(b) $B_{j}(i, k)$ represents the one-step upward transition rate from state $(i, j)$ to state $(k, j+1)(0 \leq i, k \leq c ; j=0,1, \ldots)$, which is caused by the arrival of a request when all servers are busy (i.e., when $i=c$ ), thus increasing $\neg(t)$ by 1 . Matrix $B_{j}$ ( $B$, since it is $j$-independent) is of size $(c+1) \times(c+1)$ with elements $B_{j}(i, k)$. The only nonzero element of $B_{j}$ is $B_{j}(c, c)=\lambda$. Thus, we get

$$
B_{j}=B=\left[\begin{array}{ccccccc}
0 & 0 & 0 & \ldots & 0 & 0 & 0 \\
0 & 0 & 0 & \ldots & 0 & 0 & 0 \\
\vdots & \vdots & \vdots & \vdots & \vdots & \vdots & \vdots \\
0 & 0 & \ldots & 0 & 0 & 0 \\
0 & 0 & \ldots & 0 & 0 & \lambda
\end{array}\right], \forall j \geq 0
$$

(c) $C_{j}(i, k)$ is the transition rate from state $(i, j)$ to state $(k, j-1)(0 \leq$ $i, k \leq c ; j=1,2, \ldots)$, which is due to the successful retrial of a request from the orbit. Matrix $C_{j}$ is of size $(c+1) \times(c+1)$ with its elements $C_{j}(i, k)$. The nonzero elements of $C_{j}(j \geq 1)$ are $C_{j}(i, i+1)=j \mu_{r}$ for $i=0, \ldots, c$ and $C_{j}(c, c)=j \mu_{r} P_{i m}$. Matrix $C_{j}(\forall j \geq 1)$ with elements $C_{j}(i, k)$ is written as

$$
C_{j}=\left[\begin{array}{lllllll}
0 & j \mu_{r} & 0 & \ldots & 0 & 0 & 0 \\
0 & 0 & j \mu_{r} & \ldots & 0 & 0 & 0 \\
\vdots & \vdots & \vdots & \vdots & \vdots & \vdots & \vdots \\
0 & 0 & & \ldots & 0 & 0 & j \mu_{r} \\
0 & 0 & & \ldots & 0 & 0 & j \mu_{r} P_{i m}
\end{array}\right], \forall j \geq 1
$$


Note that $C_{0}=0$ by definition.

Let $D^{A}, D^{C}$ and $D^{C_{j}}, j \geq 1$ denote diagonal matrices with the diagonal elements $D^{A}(i, i)=\sum_{k=0}^{c} A(i, k), D^{C}(i, i)=\sum_{k=0}^{c} C(i, k)$ and $D^{C_{j}}(i, i)=$ $\sum_{k=0}^{c} C_{j}(i, k)$ for $i=0, \ldots, c$. The balance equations, which equate the probability fluxes from and to the states of CTMC $Y$, and the normalization equation pertaining to CTMC $Y$ can be written as follows (see $[3,8]$ ):

$$
\begin{gathered}
\mathbf{v}_{0} Q_{1}^{(0)}+\mathbf{v}_{1} Q_{2}^{(1)}=\mathbf{0}, \\
\mathbf{v}_{j-1} Q_{0}^{(j-1)}+\mathbf{v}_{j} Q_{1}^{(j)}+\mathbf{v}_{j+1} Q_{2}^{(j+1)}=\mathbf{0}(j \geq 1), \\
\sum_{j=0}^{\infty} \mathbf{v}_{j} \mathbf{e}^{T}=1.0 \text { (normalization) }
\end{gathered}
$$

where $Q_{0}^{(j)}=B, j \geq 0 ; Q_{1}^{(j)}=A-D^{A}-B-D^{C_{j}}, j \geq 0 ; Q_{2}^{(j)}=C_{j}, j \geq 1$ and $\mathbf{e}$ is the row vector of size $c+1$ with each element equal to unity.

Using the similar argument as in $[3-5,8]$, the infinitesimal generator matrix $[15,16,21]$ of $Y$, that satisfies $\left[\mathbf{v}_{0}, \mathbf{v}_{1}, \ldots\right] Q_{Y}=\mathbf{0}$, can be constructed from equations (1) and (2) as follows:

$$
Q_{Y}=\left[\begin{array}{lllllllll}
Q_{1}^{(0)} & Q_{0}^{(0)} & 0 & \ldots & \ldots & \ldots & \ldots & \ldots & \ldots \\
Q_{2}^{(1)} & Q_{1}^{(1)} & Q_{0}^{(1)} & 0 & \ldots & \ldots & \ldots & \ldots & \ldots \\
0 & Q_{2}^{(2)} & Q_{1}^{(2)} & Q_{0}^{(2)} & \ldots & \ldots & \ldots & \ldots & \ldots \\
0 & 0 & Q_{2}^{(3)} & Q_{1}^{(3)} & Q_{0}^{(3)} & 0 & \ldots & \ldots & \ldots \\
\vdots & \vdots & \vdots & \vdots & \vdots & \vdots & \vdots & \vdots & \vdots \\
\cdots & \cdots & \cdots & Q_{2}^{(j)} & Q_{1}^{(j)} & Q_{0}^{(j)} & \ldots & \ldots & \\
\cdots & \cdots & \cdots & \cdots & Q_{2}^{(j+1)} & Q_{1}^{(j+1)} & Q_{0}^{(j+1)} & \ldots & \ldots \\
\cdots & \cdots & \cdots & \cdots & \cdots & Q_{2}^{(j+2)} & Q_{1}^{(j+2)} & Q_{0}^{(j+2)} & \ldots \\
\vdots & \vdots & \vdots & \vdots & \vdots & \vdots & \vdots & \vdots & \vdots
\end{array}\right] .
$$

It is clear that $Q_{Y}$ is a block tridiagonal matrix with

- $Q_{Y}(j, j+1)=Q_{0}^{(j)}, j \geq 0$, in the upper diagonal,

- $Q_{Y}(j, j)=Q_{1}^{(j)}, j \geq 0$ in the main diagonal,

- $Q_{Y}(j, j-1)=Q_{2}^{(j)}, j \geq 1$ in the lower diagonal.

\subsection{An Approximation}

Domenech-Benlloch et al. [20] suggested that the $\mathrm{M} / \mathrm{M} / \mathrm{c}$ retrial queue with impatient customers can be approximated by the solution of the modified multiserver retrial queue with the retrial rate

$$
\mu_{r}(j)=\left\{\begin{array}{ll}
j \mu_{r} & \text { if } j<N \\
M(N) \mu_{r} & \text { if } j \geq N
\end{array},\right.
$$


where $M(N)=E[J \mid J \geq N]$ is the conditional mean number of customers.

As a consequence, the modified multiserver retrial queue is described by a CTMC $\left.Z=\left\{I_{Z}(t),\right\rceil_{Z}(t)\right\}$ with state space $\{0,1, \ldots, c\} \times\{0,1, \ldots\}$, where $I_{Z}(t)$ represents the number of occupied servers at time $t$ and $T_{Z}(t)$ is the number of clients in the orbit waiting for retrial at time $t$. The steady-state probabilities of CTMC $Z$ are denoted by $\left.\tilde{\pi}_{i, j}=\lim _{t \rightarrow \infty} \operatorname{Pr}\left(\beth_{Z}(t)=i,\right\rceil_{Z}(t)=j\right)$, $j \geq 0,0 \leq i \leq c$, and the row vectors $\widetilde{\mathbf{v}}_{j}=\left[\widetilde{\pi}_{0, j}, \ldots, \widetilde{\pi}_{c, j}\right], j \geq 0$.

We define the transition rate matrices associated with CTMC $Z$ as $\widetilde{A}_{j}, \widetilde{A}, \widetilde{B}_{j}$, $\widetilde{B}, \widetilde{C}_{j}$ and $\widetilde{C}$ for $j \geq 0$. Note that we have $\widetilde{A}_{j}=\widetilde{A}=A$ and $\widetilde{B}_{j}=\widetilde{B}=B$ for $j \geq 0$. Furthermore, $\widetilde{C}_{j}=C_{j}$ for $0 \leq j<N$ and

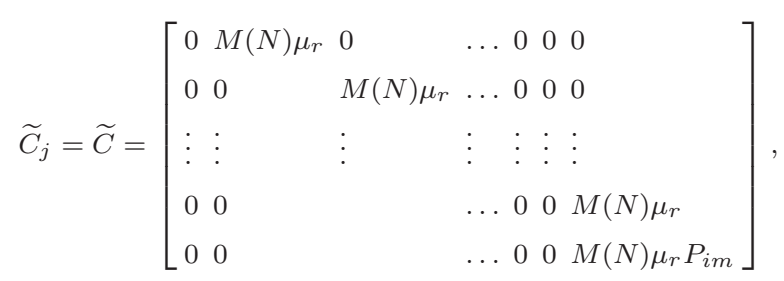

$\forall j \geq N$.

For $j \geq N$, the balance equation of CTMC $Z$ can be rewritten as

$$
\widetilde{\mathbf{v}}_{j-1} \widetilde{Q}_{0}+\widetilde{\mathbf{v}}_{j} \widetilde{Q}_{1}+\widetilde{\mathbf{v}}_{j+1} \widetilde{Q}_{2}=\mathbf{0}(j \geq N),
$$

where $\widetilde{Q}_{0}=\widetilde{B}, \widetilde{Q}_{1}=\widetilde{A}-D^{\widetilde{A}}-\widetilde{B}-D^{\widetilde{C}}, \widetilde{Q}_{2}=\widetilde{C}$.

The coefficient matrices in the difference equations (3) are $j$-independent. This leads to the following solution based on the MGM (see [16])

$$
\widetilde{\mathbf{v}}_{j}=\widetilde{\mathbf{v}}_{N-1} R^{j-N+1}(j \geq N-1),
$$

where $R$ is the unique minimal nonnegative solution of the quadratic matrix equation $\widetilde{Q}_{0}+R \widetilde{Q}_{1}+R^{2} \widetilde{Q}_{2}=0$ (see $\left.[15,16]\right)$. After the computation of $R$, the rate matrix, the steady-state probabilities for states $0 \leq j \leq N-1$ can be determined by solving the balance equations pertaining to the levels $0 \leq j<N$ and the normalization equation. 


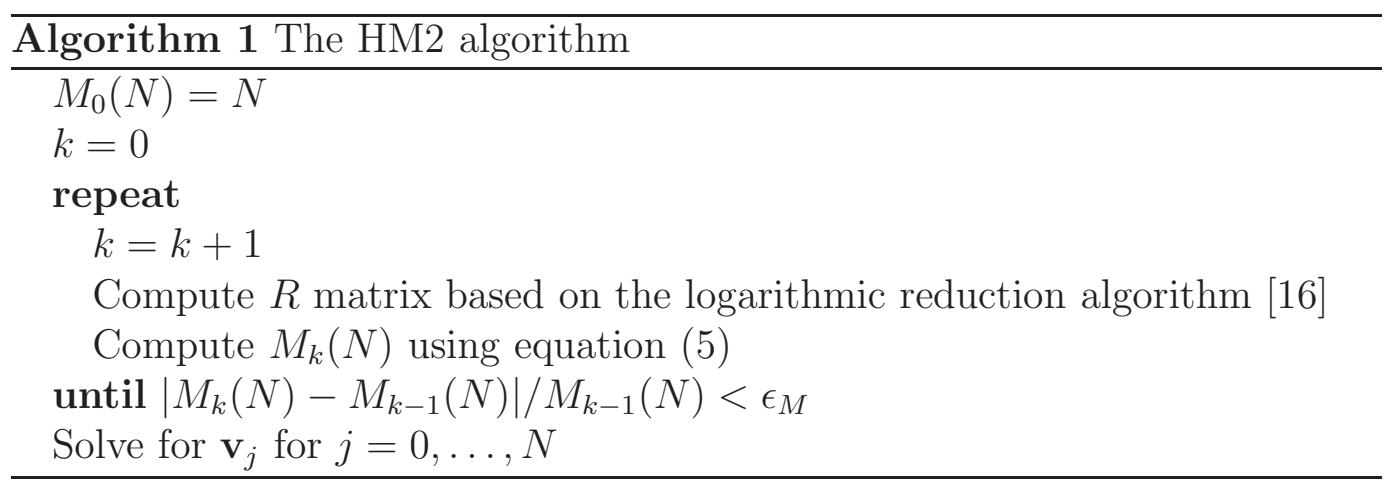

Because $R$ and $\widetilde{\mathbf{v}}_{N}$ depend on $M(N)$, we can get the fixed-point iteration

$$
M(N)=\frac{\sum_{j=N}^{\infty} j \widetilde{\mathbf{v}}_{j} \mathbf{e}}{\sum_{j=N}^{\infty} \widetilde{\mathbf{v}}_{j} \mathbf{e}}=\frac{\widetilde{\mathbf{v}}_{N}\left[R(\mathbf{I}-R)^{-1}+N \mathbf{I}\right](\mathbf{I}-R)^{-1} \mathbf{e}}{\widetilde{\mathbf{v}}_{N}(\mathbf{I}-R)^{-1} \mathbf{e}},
$$

where $\mathbf{I}$ is the identity matrix of size $(c+1) \times(c+1)$. Hence, Domenech-Benlloch et al. proposed Algorithm 1 (called HM2) in [20].

\section{An Enhanced Algorithm}

The stationary distribution of CTMC $Y$ is approximated by the steady-state probabilities of CTMC $Z$. Therefore, we need to compute the following quantities associated with CTMC Z:

- the rate matrix $R$,

- the conditional mean number of customers $M(N)$,

- the steady-state probabilities for states $0 \leq j \leq N-1$,

- the estimation of $N$.

Note that $R$ can be computed by the original algorithm of the MGM [15] and further improved algorithms of MGM $[16,18,19]$. However, the time complexity of these algorithms is $O\left(c^{3}\right)$.

In what follows, we provide a method to compute the rate matrix (Theorem 1) in Section 3.1. We derive the exact and simplified formula for the computation of the conditional mean number of customers in the orbit (Corollary 1). As a consequence, we can compute the rate matrix $R$ and the conditional mean number $M(N)$ of customers in a very efficient way. We provide a method to 
determine the steady-state probabilities for states $0 \leq j \leq N-1$ in Section 3.2. We provide the new formulae of performance measures and the relation between performance measures in Section 3.3. Next, we present our new result and our algorithm for the computation of the initial value of $N$ in Section 3.4.

\subsection{The computation of matrix $R$ and $M(N)$}

In Theorem 1 we prove that the characteristic matrix polynomial has only a single non-zero eigenvalue, and it can be computed using the bisection method. As a consequence, the rate matrix $R$ has a special form and a method can be constructed to compute the rate matrix $R$ with the computational complexity of $O(c)$. The property that the characteristic matrix polynomial has only a single non-zero eigenvalue allows the derivation of an exact equation for the conditional mean number of customers $M(N)$.

Theorem 1 The rate matrix $R$ has all rows of elements equal to zero except the last row $\mathbf{r}=\left[r_{0}, r_{1}, \ldots, r_{c}\right]$, where $r_{c}=x_{c}$ is the single eigenvalue of characteristic matrix polynomial $Q(x, M(N))=\widetilde{Q}_{0}+\widetilde{Q}_{1} x+\widetilde{Q}_{2} x^{2}$ in the interval $(0,1)$ (the corresponding left-eigenvector is $\boldsymbol{\psi}_{c}=\left[\psi_{c, 0}, \psi_{c, 2}, \ldots, \psi_{c, c}\right]$ with $\left.\psi_{c, c}=1\right)$ and $r_{i}=x_{c} \psi_{c, i}$ for $0 \leq i<c$. The computational complexity for $r_{c}$ and $\boldsymbol{\psi}_{c}$ is $O(c)$.

Proof. The steady-state probabilities of the CTMC $Z$ are expressed as

$$
\widetilde{\mathbf{v}}_{j}=\sum_{k=0}^{c} b_{k} \boldsymbol{\psi}_{k} x_{k}^{j-N+1} \quad(j \geq N-1),
$$

where $b_{k}$ are suitable coefficients to be determined using the balance equations pertaining to rows 0 to $N-1$ and the normalization equation, $\left(x_{k}, \boldsymbol{\psi}_{k}\right)$, $k=0, \ldots, c$ are the left eigenvalue-eigenvector pairs of $Q(x, M(N))=$ $\widetilde{Q}_{0}+\widetilde{Q}_{1} x+\widetilde{Q}_{2} x^{2}$ inside the unit circle. They satisfy, $\boldsymbol{\psi}_{k} Q\left(x_{k}, M(N)\right)=$ $\mathbf{0} ; \operatorname{det}\left[Q\left(x_{k}, M(N)\right)\right]=0, k=0, \ldots, c$.

Since the $(c+1) \times(c+1)$ tri-diagonal matrix $Q(x, M(N))$ can be expressed

$$
\left[\begin{array}{cccccc}
q_{1,1}(x) & q_{1,2}(x) & 0 & \ldots & 0 & 0 \\
q_{2,1}(x) & q_{2,2}(x) & q_{2,3}(x) & \ldots & 0 & 0 \\
0 & q_{3,2}(x) & q_{3,3}(x) & \ldots & 0 & 0 \\
\vdots & \vdots & \vdots & \vdots & \vdots & \vdots \\
0 & 0 & \ldots & q_{c, c-1}(x) & q_{c, c}(x) & q_{c, c+1}(x) \\
0 & 0 & \ldots & 0 & q_{c+1, c}(x) & q_{c+1, c+1}(x)
\end{array}\right],
$$


where

$$
\begin{aligned}
q_{1,1}(x)= & -\left(\lambda+M(N) \mu_{r}\right) x, \\
q_{i, i}(x)= & -\left(\lambda+M(N) \mu_{r}+(i-1) \mu\right) x \\
& (i=2, \ldots, c), \\
q_{c+1, c+1}(x)= & \lambda-\left(\lambda+c \mu+M(N) \mu_{r} P_{i m}\right) x \\
& +M(N) \mu_{r} P_{i m} x^{2}, \\
q_{i, i+1}(x)= & \lambda x+M(N) \mu_{r} x^{2} \quad(i=1, \ldots, c), \\
q_{i+1, i}(x)= & \mu i x \quad(i=1, \ldots, c) .
\end{aligned}
$$

It is easy to verify that $Q(x, M(N))$ has $c$ zero-eigenvalues. Let the nulleigenvalues be $x_{0}, \ldots, x_{c-1}$ with corresponding independent left-eigenvectors $\boldsymbol{\psi}_{0}=[1,0, \ldots, 0], \boldsymbol{\psi}_{2}=[0,1,0, \ldots, 0], \ldots, \boldsymbol{\psi}_{c-1}=[0,0, \ldots, 1,0]$, respectively As a consequence, $Q(x, M(N))$ should have a single non-zero eigenvalue $x_{c}$ strictly inside the unit disk to ensure that the stationary distribution of CTMC $\tilde{Y}$ exists.

Let $L(x, M(N))$ and $U(x, M(N))$ denote the component matrices in the LU decomposition of $Q(x, M(N))=L(x, M(N)) U(x, M(N))$ for any specific value $x$. Due to the tri-diagonal structure, the component matrices of the LU decomposition of $Q(x, M(N))$ can be written as follows

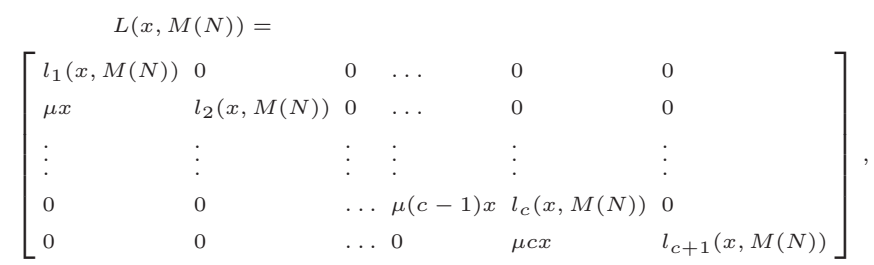

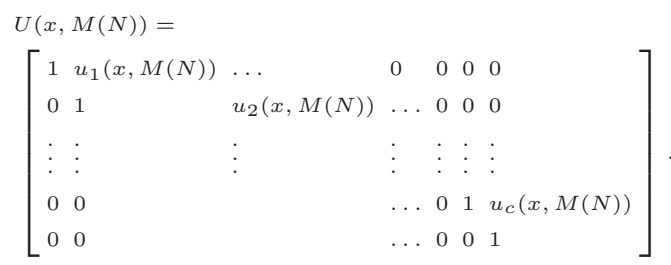

By equating the corresponding elements of $Q(x, M(N))$ and $L(x, M(N))$. $U(x, M(N))$, and using some algebraic simplifications, we get 


$$
\begin{array}{r}
l_{1}(x, M(N))=q_{1,1}(x)=-\left(\lambda+M(N) \mu_{r}\right) x, \\
l_{i}(x, M(N))+\mu(i-1) x u_{i-1}(x, M(N))=q_{i, i}(x), \\
(i=2, \ldots, c+1), \\
l_{i}(x, M(N)) u_{i}(x, M(N))=\lambda x+M(N) \mu_{r} x^{2}, \\
(i=1, \ldots, c) .
\end{array}
$$

Based on the elementary rules of matrix algebra, we obtain

$$
\begin{gathered}
\operatorname{Det}[Q(x, M(N))]=\operatorname{Det}[L(x, M(N))] \operatorname{Det}[U(x, M(N))] \\
=\prod_{i=1}^{c+1} l_{i}(x, M(N)) .
\end{gathered}
$$

From equations (7),(8) and (9), it can be verified that $l_{i}\left(x_{c}, M(N)\right) \neq 0(1 \leq$ $i \leq c)$. Hence, $\operatorname{Det}\left[Q\left(x_{c}, M(N)\right)\right]=0$ (from equation (10)) gives rise to $l_{c+1}\left(x_{c}, M(N)\right)=0$. This means $x_{c}$ is the root of $l_{c+1}(x, M(N))$ in the interval $(0,1)$. The bisection method [22] can be applied to find the root of $l_{c+1}(x, M(N))$ in the interval $(0,1)$.

Since $\left(x_{c}, \boldsymbol{\psi}_{c}\right)$ are left eigenvalue-eigenvector pair, we can write

$$
\begin{aligned}
\boldsymbol{\psi}_{c} Q\left(x_{c}, M(N)\right) & =\mathbf{0}, \\
\boldsymbol{\psi}_{c} L\left(x_{c}, M(N)\right) U\left(x_{c}, M(N)\right) & =\mathbf{0}, \\
\boldsymbol{\psi}_{c} L\left(x_{c}, M(N)\right) U\left(x_{c}, M(N)\right) U\left(x_{c}, M(N)\right)^{-1} & = \\
\mathbf{0} U\left(x_{c}, M(N)\right)^{-1}, &
\end{aligned}
$$

because $U\left(x_{c}, M(N)\right)$ is non-singular,

$$
\boldsymbol{\psi}_{c} L\left(x_{c}, M(N)\right)=\mathbf{0} .
$$

Expanding equation (11) we obtain the recursive relations $\psi_{c, i}=\frac{-(i+1) \mu x_{c} \psi_{c, i+1}}{l_{i+1}\left(x_{c}\right)}$ between $\psi_{c, i}$ and $\psi_{c, i+1}$, for $i=c-1, \ldots, 0$.

There are a number of eigenvectors corresponding to the same eigenvalue, but the ratio of the elements in these eigenvectors does not change. Applying this property, we can determine $\boldsymbol{\psi}_{c}=\left[\psi_{c, 0}, \psi_{c, 1}, \ldots, \psi_{c, c}\right]$ by setting $\psi_{c, c}=1$ and using the above recursive relations and equations (7),(8), (9), to compute $\psi_{c, i}$ for $i=c-1, \ldots, 0$.

From (4) and (6) we get $R=\Psi^{-1} \cdot \operatorname{diag}\left(0,0, \ldots, 0, x_{c}\right) \cdot \Psi$, where $\Psi=$ $\left[\psi_{0}, \psi_{1}, \ldots, \psi_{c}\right]^{T}$. Therefore, the rate matrix $R$ has all rows of elements equal 
to zero except the last row $\mathbf{r}=\left[r_{0}, r_{1}, \ldots, r_{c}\right]$. Furthermore, we get $r_{i}=x_{c} \psi_{c, i}$ for $0 \leq i<c$ and $r_{c}=x_{c}$ after a simple algebraic step.

The complexity for computing $r_{c}$ is $O(c)$ as the consequence of the following facts: (i) the last element $l_{c+1}(x, M(N))$ in the main diagonal of $L(x, M(N))$ can be determined after $c+1$ steps due to the tri-diagonal structure of $Q(x, M(N))$; (ii) the number of iterations in the bisection method for finding a root in the interval $(0,1)$ to achieve the solution tolerance $\epsilon_{r}$ is $\log _{2}\left(1 / \epsilon_{r}\right)$ (see $[22])$.

Corollary 1 The conditional mean value $M(N)=E[J \mid J \geq N]$ of the number $J$ of customers in the orbit under the condition $J \geq N$ can be expressed in the following closed-form:

$$
M(N)=N-1+\frac{1}{1-r_{c}}=N+\frac{r_{c}}{1-r_{c}} .
$$

Proof. The consequence of Theorem 1 and (4) is

$$
\begin{gathered}
\widetilde{\mathbf{v}}_{N}=\widetilde{\mathbf{v}}_{N-1} R=\tilde{\pi}_{c, N-1} \mathbf{r}, \\
\mathbf{r} R=r_{c} \mathbf{r}, \quad R^{2}=r_{c} R .
\end{gathered}
$$

Substituting (13) to (5) we obtain

$$
\begin{aligned}
M= & \frac{\widetilde{\pi}_{c, N-1} \mathbf{r}\left[R(\mathbf{I}-R)^{-1}+N \mathbf{I}\right](\mathbf{I}-R)^{-1} \mathbf{e}}{\tilde{\pi}_{c, N-1} \mathbf{r}(\mathbf{I}-R)^{-1} \mathbf{e}} \\
= & \frac{\mathbf{r}\left[R(\mathbf{I}-R)^{-1}+N \mathbf{I}\right](\mathbf{I}-R)^{-1} \mathbf{e}}{\mathbf{r}(\mathbf{I}-R)^{-1} \mathbf{e}}
\end{aligned}
$$

Since $R$ has all rows with zero-elements except the last row, the rank of $R$ is 1 . Applying the result of [23], we can write

$$
(\mathbf{I}-R)^{-1}=\mathbf{I}-\frac{\mathbf{I}(-R) \mathbf{I}}{1-\operatorname{tr}(R \mathbf{I})}=\mathbf{I}+\frac{R}{1-r_{c}},
$$

where the trace $\operatorname{tr}(R \mathbf{I})$ of matrix $R \mathbf{I}$ is the sum of all the elements on the main diagonal of matrix $R \mathbf{I}$. Substituting (16) into (15) and utilizing (14) yields (12) after some algebraic steps.

Corollary 1 expresses that the conditional mean value $M(N)=E[J \mid J \geq N]$ of the number $J$ of customers in the orbit under the condition $J \geq N$ is the simple function of the single eigenvalue and $N$. Note that this result is the direct consequence of Theorem 1. 


\subsection{The computation of the steady-state probabilities}

The task is to compute the steady-state probabilities for states $0 \leq j \leq N-1$ by solving the balance equations pertaining to the levels $0 \leq j<N$ and the normalization equation.

Let us introduce auxiliary variables $u_{i, j}=\tilde{\pi}_{i, j} / \widetilde{\pi}_{c, N-1}$ and $\mathbf{u}_{j}=\left[u_{0, j}, \ldots, u_{c, j}\right]$, $j \geq 0$. Hence $u_{c, N-1}=1$. Note that $\widetilde{\pi}_{-1, j}=u_{-1, j}=0$ by definition.

From (4), we have $\widetilde{\mathbf{v}}_{N}=\widetilde{\mathbf{v}}_{N-1} R$. Thus, $\widetilde{\pi}_{i, N}=r_{i} \widetilde{\pi}_{c, N-1}$ for $i=0, \ldots, c$. Using the relation between $\widetilde{\pi}_{c, N-1}$ and $\widetilde{\pi}_{i, N}$, we can write the balance equation for level $N-1$ as

$$
\begin{array}{r}
\left(\lambda+i \mu+(N-1) \mu_{r}\right) \widetilde{\pi}_{i, N-1} \\
=\lambda \widetilde{\pi}_{i-1, N-1}+M(N) \mu_{r} \widetilde{\pi}_{i-1, N}+(i+1) \mu \widetilde{\pi}_{i+1, N-1} \\
=\lambda \widetilde{\pi}_{i-1, N-1}+M(N) \mu_{r} r_{i-1} \widetilde{\pi}_{c, N-1}+(i+1) \mu \widetilde{\pi}_{i+1, N-1}, \\
0 \leq i<c .
\end{array}
$$

$$
\begin{array}{r}
\left(\lambda+c \mu+(N-1) \mu_{r} P_{i m}\right) \widetilde{\pi}_{c, N-1} \\
=\lambda \widetilde{\pi}_{c-1, N-1}+M(N) \mu_{r} \widetilde{\pi}_{c-1, N} \\
+\lambda \widetilde{\pi}_{c, N-2}+M(N) \mu_{r} P_{i m} \widetilde{\pi}_{c, N} \\
=\lambda \widetilde{\pi}_{c-1, N-1}+M(N) \mu_{r} r_{c-1} \widetilde{\pi}_{c, N-1} \\
+\lambda \widetilde{\pi}_{c, N-2}+M(N) \mu_{r} r_{c} P_{i m} \widetilde{\pi}_{c, N-1} .
\end{array}
$$

Therefore, we obtain

$$
\begin{gathered}
\left(\lambda+i \mu+(N-1) \mu_{r}\right) u_{i, N-1} \\
=\lambda u_{i-1, N-1}+M(N) \mu_{r} r_{i-1}+(i+1) \mu u_{i+1, N-1}, \\
0 \leq i<c . \\
\left(\lambda+c \mu+(N-1) \mu_{r} P_{i m}\right) \\
=\lambda u_{c-1, N-1}+M(N) \mu_{r} u_{c-1, N} \\
+\lambda u_{c, N-2}+M(N) \mu_{r} P_{i m} u_{c, N} \\
=\lambda u_{c-1, N-1}+M(N) \mu_{r} r_{c-1} \\
+\lambda u_{c, N-2}+M(N) \mu_{r} r_{c} P_{i m} .
\end{gathered}
$$

Expanding the balance equation pertaining to level $j, 0 \leq j<N-1$, we get

$$
\begin{aligned}
\left(\lambda+i \mu+j \mu_{r}\right) \tilde{\pi}_{i, j}= & \lambda \widetilde{\pi}_{i-1, j} \\
& +(j+1) \mu_{r} \widetilde{\pi}_{i-1, j+1}+(i+1) \mu \widetilde{\pi}_{i+1, j}, \\
& 0 \leq i<c, 0 \leq j<N-1,
\end{aligned}
$$




$$
\begin{aligned}
& \left(\lambda+c \mu+j \mu_{r} P_{i m}\right) \tilde{\pi}_{c, j}=\lambda \widetilde{\pi}_{c-1, j} \\
& \quad+(j+1) \mu_{r} \widetilde{\pi}_{c-1, j+1}+\lambda \widetilde{\pi}_{c, j-1}+(j+1) \mu_{r} P_{i m} \widetilde{\pi}_{c, j+1}, \\
& 0 \leq j<N-1,
\end{aligned}
$$

which follows

$$
\begin{aligned}
\left(\lambda+i \mu+j \mu_{r}\right) u_{i, j}= & \lambda u_{i-1, j} \\
& +(j+1) \mu_{r} u_{i-1, j+1}+(i+1) \mu u_{i+1, j} \\
& 0 \leq i<c, 0 \leq j<N-1,
\end{aligned}
$$

$$
\begin{aligned}
& \left(\lambda+c \mu+j \mu_{r} P_{i m}\right) u_{c, j}=\lambda u_{c-1, j} \\
& +(j+1) \mu_{r} u_{c-1, j+1}+\lambda u_{c, j-1}+(j+1) \mu_{r} P_{i m} u_{c, j+1} \\
& \quad 0 \leq j<N-1 .
\end{aligned}
$$

Note that both equations (17) and (20) have the tridiagonal form if $u_{i-i, j+1}$ is determined in the previous step:

$$
\alpha_{i j} u_{i-1, j}+\beta_{i, j} u_{i, j}+\gamma_{i j} u_{i+1, j}=\omega_{i, j}
$$

for $0 \leq i \leq c-1$ and $0 \leq j \leq N-1$. Therefore, the Thomas algorithm can be used to solve the steady-state probabilities in an efficient way [24]. The adaptation of the Thomas algorithm for the present problem is presented in Algorithm 2, where line 6 is the result of equating the flow rate into and out of level $j$ of the orbit. 


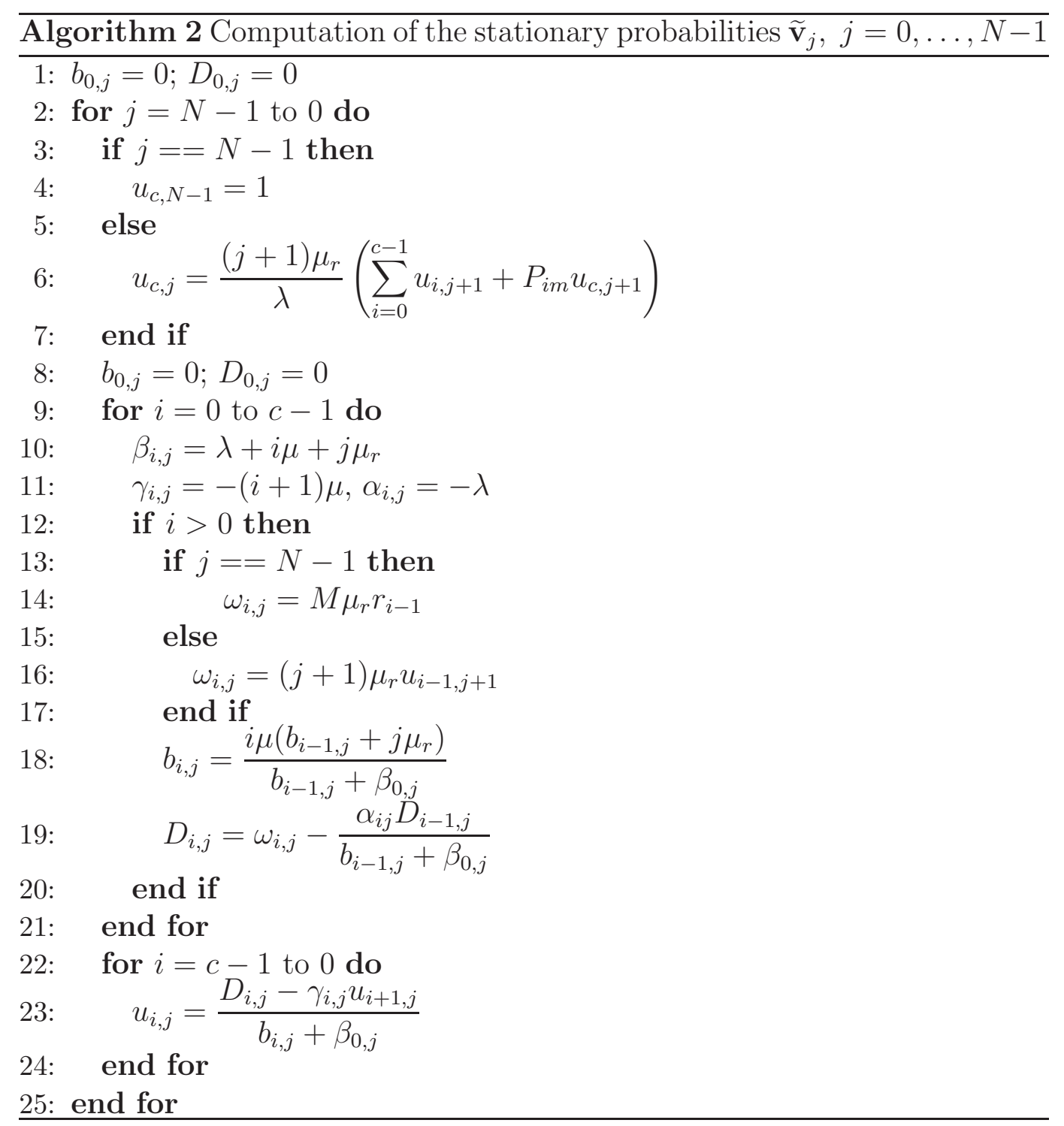

From the normalization equation $\sum_{j=0}^{N-2} \widetilde{\mathbf{v}}_{j} \mathbf{e}+\widetilde{\mathbf{v}}_{N-1}(\mathbf{I}-R)^{-1} \mathbf{e}=1$, we get

$$
\tilde{\pi}_{c, N-1}=\frac{1}{\sum_{j=0}^{N-2} \mathbf{u}_{j} \mathbf{e}+\mathbf{u}_{N-1}(\mathbf{I}-R)^{-1} \mathbf{e}}
$$

Then, we obtain

$$
\tilde{\pi}_{i, j}=u_{i, j} \tilde{\pi}_{c, N-1}=\frac{u_{i, j}}{\sum_{j=0}^{N-2} \mathbf{u}_{j} \mathbf{e}+\mathbf{u}_{N-1}(\mathbf{I}-R)^{-1} \mathbf{e}}
$$




\subsection{Performance Measures}

The blocking probability $P_{b}$, the immediate service probability $P_{i s}$, the delayed service probability $P_{d s}$, the nonservice probability $P_{n s}$ and the mean number of users in the retrial orbit $N_{\text {ret }}$ can be determined [20] as follows:

$$
\begin{aligned}
P_{b}= & \sum_{m=0}^{N-1} \widetilde{\mathbf{v}}_{m} \mathbf{z}+\widetilde{\mathbf{v}}_{N}(\mathbf{I}-R)^{-1} \mathbf{z}, \\
P_{i s}= & 1-P_{b}, \\
P_{d s}= & \lambda^{-1} \mu_{r}\left[\sum_{m=0}^{N-1} m \widetilde{\mathbf{v}}_{m} \mathbf{o}+M(N) \widetilde{\mathbf{v}}_{N}(\mathbf{I}-R)^{-1} \mathbf{o}\right], \\
P_{n s}= & \lambda^{-1} P_{i m} \mu_{r}\left[\sum_{m=0}^{N-1} m \widetilde{\mathbf{v}}_{m} \mathbf{z}+M(N)_{\mathbf{v}_{N}}(\mathbf{I}-R)^{-1} \mathbf{z}\right], \\
N_{r e t}= & \sum_{m=0}^{N-1} m \widetilde{\mathbf{v}}_{m} \mathbf{e} \\
& +\widetilde{\mathbf{v}}_{N}\left(R(\mathbf{I}-R)^{-1}+N \mathbf{I}\right)(\mathbf{I}-R)^{-1} \mathbf{e},
\end{aligned}
$$

where $\mathbf{z}=[0,0, \ldots, 0,1], \mathbf{o}=[1,1, \ldots, 1,0], \mathbf{e}=[1,1, \ldots, 1,1]$. Note that $\mathbf{e}=\mathbf{o}+\mathbf{z}$. Because $P_{i s}+P_{d s}+P_{n s}=1$ (see [20]) and $P_{i s}=1-P_{b}$, we get $P_{b}=P_{d s}+P_{n s}$.

However, the direct application of equations (22) defined in [20] is not efficient when one implements a computer program. To compute the performance measures in an efficient way, we derive simpler equations than (22) after some algebraic steps that are presented in Proposition 1, 2, 3 and 4. Note that the equations for the performance measures are utilized in Algorithm 4 (see Section 3.4).

Let us define the following quantities

$$
\begin{aligned}
\mathbf{a} & =\widetilde{\mathbf{v}}_{N}\left(R(\mathbf{I}-R)^{-1}+N \mathbf{I}\right)(\mathbf{I}-R)^{-1} \\
a_{1} & =\sum_{m=0}^{N-1} m \widetilde{\mathbf{v}}_{m} \mathbf{o}+M(N) \widetilde{\mathbf{v}}_{N}(\mathbf{I}-R)^{-1} \mathbf{o} \\
a_{2} & =\sum_{m=0}^{N-1} m \widetilde{\mathbf{v}}_{m} \mathbf{z}+M(N) \widetilde{\mathbf{v}}_{N}(\mathbf{I}-R)^{-1} \mathbf{z} .
\end{aligned}
$$

Therefore, $P_{d s}=\lambda^{-1} \mu_{r} a_{1}$. 
Proposition 1 The nonservice probability is expressed as

$$
\begin{aligned}
P_{n s} & =\lambda^{-1} P_{i m} \mu_{r} a_{2}=\lambda^{-1} P_{i m} \mu_{r} \\
& \left(\sum_{m=1}^{N-2} m \tilde{\pi}_{c, m}+\frac{(M(N)-1) \widetilde{\pi}_{c, N-1}}{1-r_{c}}\right) .
\end{aligned}
$$

\section{Proof.}

Substituting (16) to the definition of $\mathbf{a}$, we obtain

$$
\begin{aligned}
& \mathbf{a}=\widetilde{\mathbf{v}}_{N}\left(R(\mathbf{I}-R)^{-1}+N \mathbf{I}\right)(\mathbf{I}-R)^{-1} \\
& =\widetilde{\mathbf{v}}_{N}\left(R\left(\mathbf{I}+\frac{R}{1-r_{c}}\right)+N \mathbf{I}\right)(\mathbf{I}-R)^{-1} \\
& =\widetilde{\mathbf{v}}_{N}\left(\frac{R}{1-r_{c}}+N \mathbf{I}\right)(\mathbf{I}-R)^{-1} \quad(\operatorname{using}(13)) \\
& =\tilde{\pi}_{c, N-1} \mathbf{r}\left(\frac{R}{1-r_{c}}+N \mathbf{I}\right)(\mathbf{I}-R)^{-1} \quad(\operatorname{using}(14)) \\
& =\widetilde{\pi}_{c, N-1}\left(\frac{r_{c} \mathbf{r}}{1-r_{c}}+N \mathbf{r}\right)(\mathbf{I}-R)^{-1} \quad(\operatorname{using}(13)) \\
& =\widetilde{\pi}_{c, N-1} M(N) \mathbf{r}(\mathbf{I}-R)^{-1} \quad(\operatorname{using}(12)) \\
& =M(N) \widetilde{\mathbf{v}}_{N}(\mathbf{I}-R)^{-1} \quad(\text { using }(13)) \\
& =\widetilde{\pi}_{c, N-1} M(N) \mathbf{r}\left(\mathbf{I}+\frac{R}{1-r_{c}}\right) \quad(\operatorname{using}(16)) \\
& =\widetilde{\pi}_{c, N-1} M(N)\left(\mathbf{r}+\frac{r_{c} \mathbf{r}}{1-r_{c}}\right) \quad(\operatorname{using}(14)) \\
& =\frac{\tilde{\pi}_{c, N-1} M(N)}{1-r_{c}} \mathbf{r} \text {. }
\end{aligned}
$$

Substituting (28) into (25), we obtain 


$$
\begin{aligned}
a_{2} & =\sum_{m=0}^{N-1} m \widetilde{\mathbf{v}}_{m} \mathbf{z}+\mathbf{a} \mathbf{z} \\
& =\sum_{m=0}^{N-1} m \widetilde{\mathbf{v}}_{m} \mathbf{z}+\frac{\widetilde{\pi}_{c, N-1} M(N)}{1-r_{c}} \mathbf{r} \mathbf{z} \\
& =\sum_{m=1}^{N-1} m \widetilde{\pi}_{c, m}+\frac{\widetilde{\pi}_{c, N-1} M(N) r_{c}}{1-r_{c}} \\
& =\sum_{m=1}^{N-2} m \widetilde{\pi}_{c, m}+\widetilde{\pi}_{c, N-1}\left[N-1+\frac{r_{c} M(N)}{1-r_{c}}\right] \\
& =\sum_{m=1}^{N-2} m \widetilde{\pi}_{c, m}+\frac{(M(N)-1) \widetilde{\pi}_{c, N-1}}{1-r_{c}}(\text { using }(12)) .
\end{aligned}
$$

Equation (29) yields (26). $\square$

Proposition 2 The mean number of users in the retrial orbit is

$$
N_{r e t}=a_{1}+a_{2}
$$

Proof. From the definition of $a_{1}$ and $a_{2}$, we get

$$
\begin{aligned}
a_{1}+a_{2} & =\sum_{m=0}^{N-1} m \widetilde{\mathbf{v}}_{m}(\mathbf{o}+\mathbf{z})+M(N) \widetilde{\mathbf{v}}_{N}(\mathbf{I}-R)^{-1}(\mathbf{o}+\mathbf{z}) \\
& =\sum_{m=0}^{N-1} m \widetilde{\mathbf{v}}_{m} \mathbf{e}+M(N) \widetilde{\mathbf{v}}_{N}(\mathbf{I}-R)^{-1} \mathbf{e}
\end{aligned}
$$

Utilizing (27) and the definition of $N_{\text {ret }}$, we obtain equation (30).

Proposition 3 We can obtain the blocking probability $P_{b}$ as follows:

$$
P_{b}=\sum_{m=0}^{N-2} \tilde{\pi}_{c, m}+\frac{\tilde{\pi}_{c, N-1}}{1-r_{c}} .
$$

Proof. Utilizing (16), we obtain 


$$
\begin{aligned}
P_{b} & =\sum_{m=0}^{N-1} \widetilde{\mathbf{v}}_{m} \mathbf{z}+\widetilde{\mathbf{v}}_{N}(\mathbf{I}-R)^{-1} \mathbf{z} \\
& =\sum_{m=0}^{N-1} \widetilde{\mathbf{v}}_{m} \mathbf{z}+\widetilde{\mathbf{v}}_{N-1} R\left(\mathbf{I}+\frac{R}{1-r_{c}}\right) \mathbf{z} \\
& =\sum_{m=0}^{N-1} \widetilde{\mathbf{v}}_{m} \mathbf{z}+\widetilde{\mathbf{v}}_{N-1} \frac{R}{1-r_{c}} \mathbf{z} \\
& =\sum_{m=0}^{N-1} \widetilde{\mathbf{v}}_{m} \mathbf{z}+\widetilde{\pi}_{c, N-1} \frac{\mathbf{r}}{1-r_{c}} \mathbf{z} \\
& =\sum_{m=0}^{N-2} \widetilde{\mathbf{v}}_{m} \mathbf{z}+\widetilde{\mathbf{v}}_{N-1} \mathbf{z}+\widetilde{\pi}_{c, N-1} \frac{\mathbf{r}}{1-r_{c}} \mathbf{z} \\
& =\sum_{m=0}^{N-2} \widetilde{\pi}_{c, m}+\widetilde{\pi}_{c, N-1}+\widetilde{\pi}_{c, N-1} \frac{r_{c}}{1-r_{c}} \\
& =\sum_{m=0}^{N-2} \widetilde{\pi}_{c, m}+\frac{\widetilde{\pi}_{c, N-1}}{1-r_{c}} . \square .
\end{aligned}
$$

Proposition 4 The following relation exists between the performance measures

$$
N_{r e t}=\frac{\lambda}{\mu_{r}}\left(\frac{P_{n s}\left(1-P_{i m}\right)}{P_{i m}}+P_{b}\right) .
$$

Proof. From $P_{d s}=\lambda^{-1} \mu_{r} a_{1}, P_{n s}=\lambda^{-1} P_{i m} \mu_{r} a_{2}, P_{b}=P_{d s}+P_{n s}$ and $N_{r e t}=$ $a_{1}+a_{2}$, we get

$$
\begin{aligned}
N_{r e t} & =\frac{\lambda}{\mu_{r}}\left(\frac{P_{n s}}{P_{i m}}+P_{d s}\right) \\
& =\frac{\lambda}{\mu_{r}}\left(\frac{P_{n s}}{P_{i m}}+P_{b}-P_{n s}\right) \\
& =\frac{\lambda}{\mu_{r}}\left(\frac{P_{n s}\left(1-P_{i m}\right)}{P_{i m}}+P_{b}\right) .
\end{aligned}
$$

\subsection{An Estimation of $N$}

Domenech-Benlloch et al. [20] presented some numerical results concerning choosing the appropriate value of threshold $N$ to achieve the required accuracy of the approximation of performance measures. However, the authors [20] did not present a systematic way to find the appropriate value of threshold $N$. In this section, we will show an efficient method to estimate threshold $N$. 


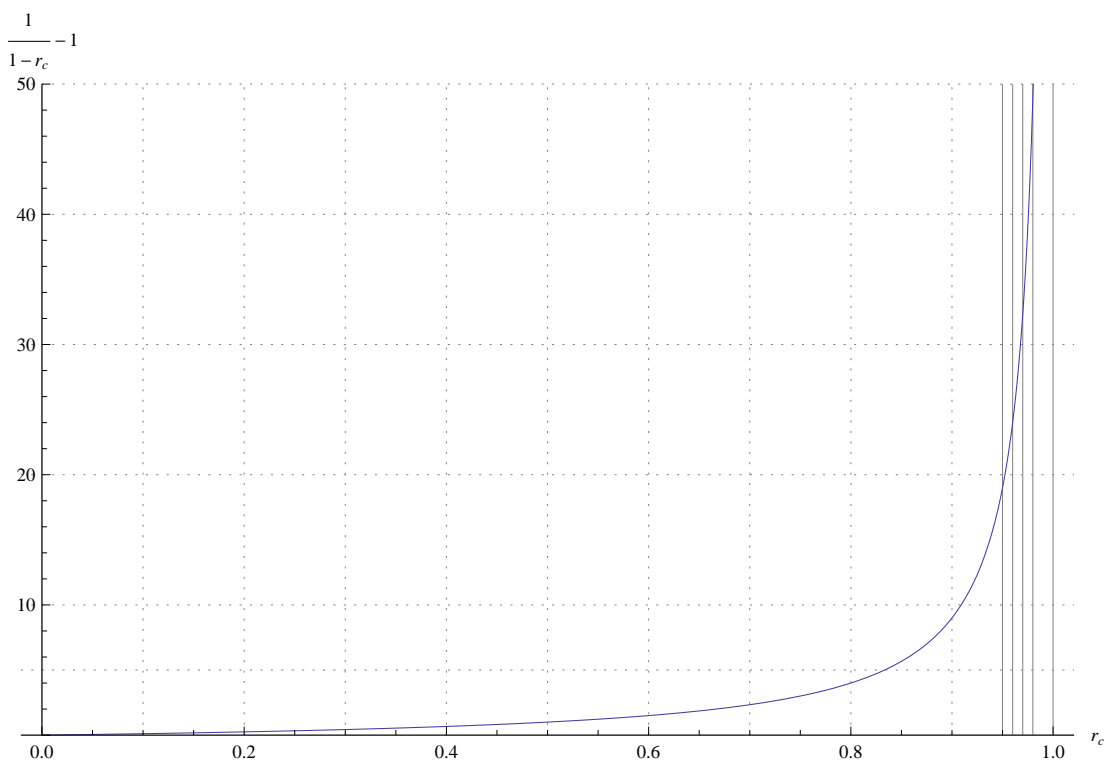

Fig. $1.1 /\left(1-r_{c}\right)-1$ vs $r_{c}$

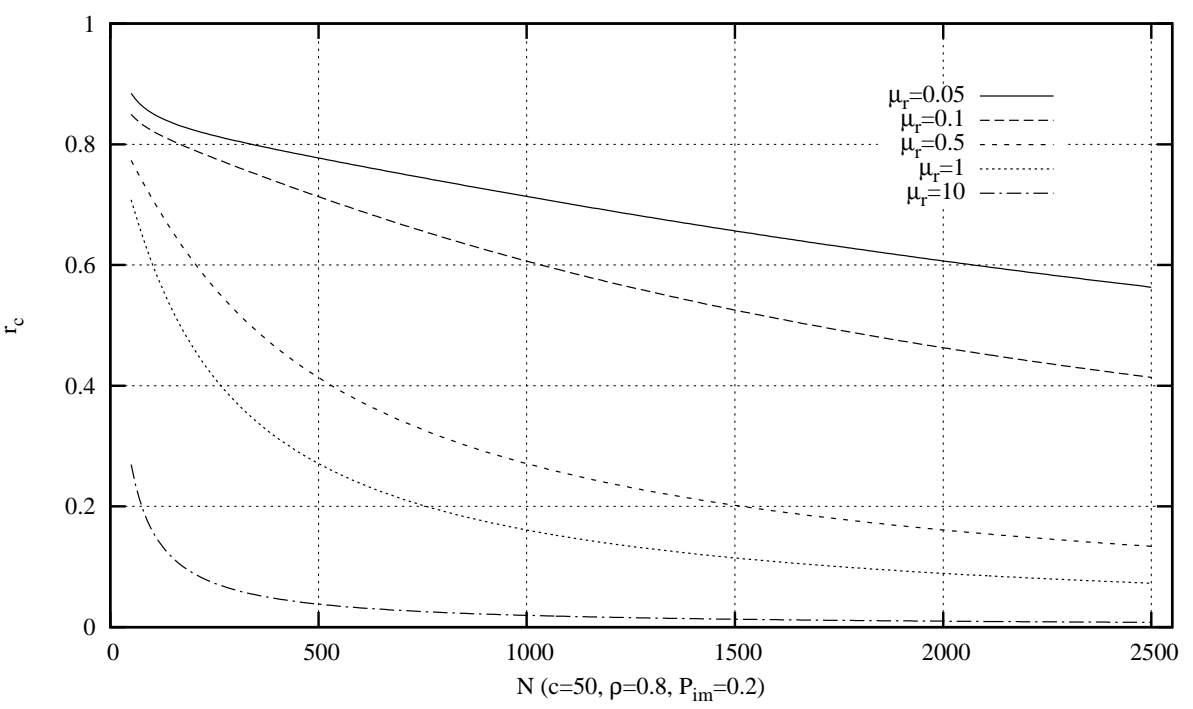

Fig. 2. $r_{c}$ vs $N$ for $c=50, \rho=0.8, P_{i m}=0.2$ and $\mu=1$

From equation (4), the tail of the distribution $\widetilde{\mathbf{v}}_{j}$ is geometrically distributed with parameter $r_{c}$. In the stable state of the system described by CTMC $\tilde{Y}$, the higher the value $N$ we choose, the smaller the value of $\mathbf{v}_{N}$ is. It is anticipated that the higher the chosen value of $N$ is the smaller the value of $r_{c}$ is if the system is in the stable state (see Figure 2).

As one observes from Figure 1, the slope of the tangent line to the curve $1 /\left(1-r_{c}\right)-1$ increases as $r_{c}$ approaches 1 , and $M(N)-N$ too. Let $r_{t h}$ be the selected upper limit of $r_{c}$ (the purpose of the selected upper limit is to search 
for the initial value of $N$ to save the computational time), i.e., $0<r_{c} \leq r_{t h}$.
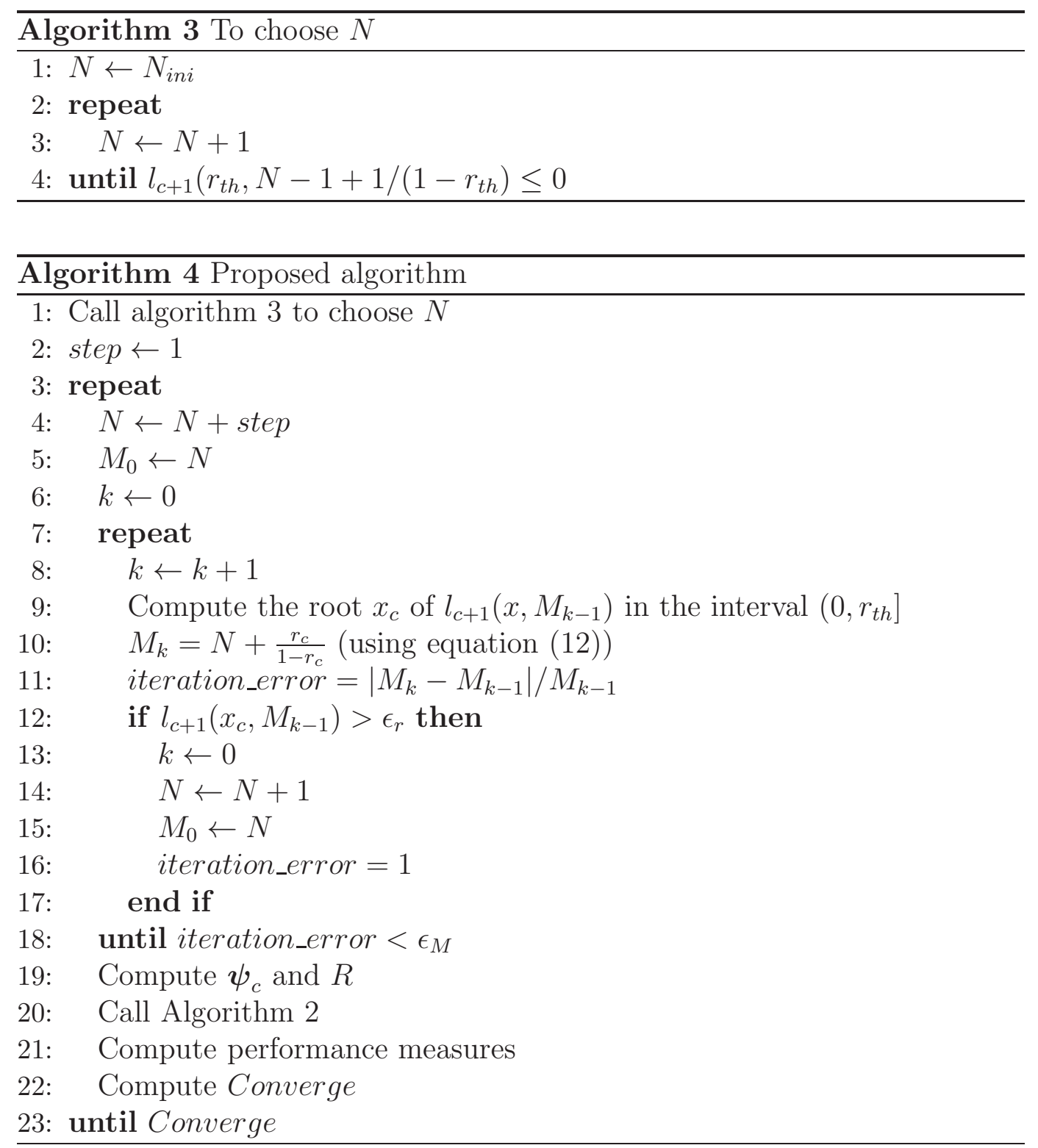

As stated before we also need to find the initial value of $N$. To save the computational time of the search for the initial value of $N$, we only check whether $l_{c+1}(x, M(N))$ has a root in the interval $\left(0, r_{t h}\right]$ instead of determining $r_{c}$. Because $l_{c+1}(0, M(N))=\lambda$ holds, we have to examine whether $l_{c+1}\left(r_{t h}, M(N)\right) \leq$ 0 in the first stage of our proposed solution. However, $M(N)$ is not known in advance. To resolve this problem, Theorem 2 is applied to choose the initial value of $N$ (see Algorithm 3), where $l_{c+1}\left(r_{t h}, N-1+1 /\left(1-r_{t h}\right)\right) \leq 0$ is verified.

Theorem 2 If $r_{c}$ is the eigenvalue of $Q(x, M(N))$ in the interval $(0,1)$, and $l_{c+1}\left(r_{t h}, N-1+1 /\left(1-r_{t h}\right)\right) \leq 0$ for $r_{t h}>0$, then $r_{c}$ is bounded by $r_{t h}$ (i.e., $\left.0<r_{c} \leq r_{t h}\right)$. 


\section{Proof.}

Assume that $r_{t h}<r_{c}$, which follows $l_{c+1}\left(r_{t h}, M(N)\right)>0$ because $r_{c}$ is the eigenvalue of $Q(x, M(N))$ in the interval $(0,1)$ (i.e., $\left.l_{c+1}\left(r_{c}, M(N)\right)=0\right)$ and $l_{c+1}(0, M(N))=\lambda$.

Note that $l_{c+1}(x, y)$ is a monotonically decreasing function with respect to $y$ for any constant $x, 0<x<1$, because

- $l_{c+1}(0, y)=\lambda$ holds,

- $l_{c+1}(x, y)$ has a single root with respect to $x$ in the interval $(0,1)$ for any constant $y$, and

- the higher the value of $y$ is the smaller the root with respect to $x$ is.

We have $N-1+1 /\left(1-r_{t h}\right)<M(N)=N-1+1 /\left(1-r_{c}\right)$ from the assumption. Therefore, $l_{c+1}\left(r_{t h}, N-1+1 /\left(1-r_{t h}\right)\right)>l_{c+1}\left(r_{t h}, M(N)\right)$.

Using $l_{c+1}\left(r_{t h}, M(N)\right)>0$, we obtain $l_{c+1}\left(r_{t h}, N-1+1 /\left(1-r_{t h}\right)\right)>0$, which contradicts the given condition $l_{c+1}\left(r_{t h}, N-1+1 /\left(1-r_{t h}\right)\right) \leq 0$. Therefore, $r_{t h}<r_{c}$ does not hold, which yields that $r_{c}$ is bounded by $r_{t h}$.

Theorem 2 is used in our proposed Algorithm 4 to find the initial value of $N$.

\subsection{The Convergence Criterion of a Proposed Algorithm}

We present the details of a proposed computational procedure in Algorithm 4 that integrates key results in Section 3.1-3.4. In Algorithm 4 two loops are applied after the initial choice of $N$. The inner loop is needed to find $M(N)$ of a certain value $N$, while the outer loop is to tune $N$ to obtain the required accuracy of the estimation of performance measures. A notable feature of the proposed procedure compared to the HM2 algorithm is that the computation of $M(N)$ does not require the computation of the steady-state probabilities. Furthermore, the algorithm is enhanced with the computation of threshold $N$.

To define the convergence criterion Converge we made the following investigation: we compute the performance measures by running the core (between lines 4 and 19 of Algorithm 3) of our algorithm with fixing $N$ for parameters $\mu=1 / 180, \mu_{r}=0.01, P_{i m}=0.2, \epsilon_{M}=10^{-3}$ and $\epsilon_{r}=10^{-10}$ (Figures 3 and 4 ). From Figures 3 and 4 the performance measures converges as $N$ grows. Furthermore, a high oscillation is observed in Figure 4 as well (see the e-companion for more numerical results with other settings of parameters). Therefore, the convergence criterion (to determine when the performance measures reach the "stable state") is defined as the relative error between two moving averages 
concerning a specific performance measure

$$
\text { Converge }:=\frac{\left|\sum_{i=L}^{K} \chi_{i} /(K-L+1)-\sum_{i=1}^{K} \chi_{i} / K\right|}{\sum_{i=L}^{K} \chi_{i} /(K-L+1)}<\epsilon_{p},
$$

where $\chi_{i}$ 's, $i=1, \ldots, K$, are the latest values of a chosen performance measure (e.g., $N_{\text {ret }}$ ) determined so far by the algorithm, $\epsilon_{p}$ is the specified accuracy, $K$ and $L$ are parameters. It is obvious that the minimum choice of $K$ and $L$ is $K=3$ and $L=2$. Furthermore, the higher the values of $K$ and $L$ are, the more time is needed, but the better the guarantee of convergence is ensured.

In Table 1 we summarize the computational time of the algorithm for $\rho=$ $\lambda /(\mu c), \mu=1 / 180, \mu_{r}=0.01, P_{i m}=0.2$. As observed the algorithm successfully stops in the stable region of performance measures (see Figures 3 and 4). From numerical results (see the e-companion for results with other settings of $K$ and $L$ ), the minimum choice of $K=3$ and $L=2$ can guarantee that the proposed algorithm steps over the "oscillation period". Results in Table 1 and the e-companion empirically show that $P_{n s}$ can be set as the main performance measure in the convergence criterion Converge for determining all performance measures.

\section{Computational Times of the Proposed Algorithm}

We plot the computational time versus $c$ and $N$ in Figures 5 and 6 , on a machine with Intel ${ }^{\circledR}$ Core $^{\mathrm{TM}} 2$ Duo T9400 $2.53 \mathrm{GHz}$ processor (note that the algorithm is implemented in Mathematica) for parameters $\rho=\lambda /(\mu c)=0.8$, $\mu=1, P_{i m}=0.2, \epsilon_{r}=10^{-10}, \epsilon_{M}=10^{-3}$. In the curves the computational time of the HM2 algorithm [20] and the core (between lines 4 and 19 of Algorithm 3) of our algorithm. As observed the computational time of the original algorithm HM2 is a rapidly increasing function of $c$, while the computational complexity of the core of our algorithm is only $O(c)$.

In Figure 7, we plot the computational time of our algorithm versus $\rho$ and $c$. It is observed from Table 1 and Figure 7 that $\rho$ impacts the convergence of the algorithms. The explanation behind this phenomenon is that the higher $\rho$ is the more likely the oscillation is (see Figure 4 and illustrations in the e-companion). Therefore, more computational time is needed to step over the oscillation. In other words, the algorithm needs more time to reach the convergence due to the oscillation phenomenon at large values of $\rho$. However, the 

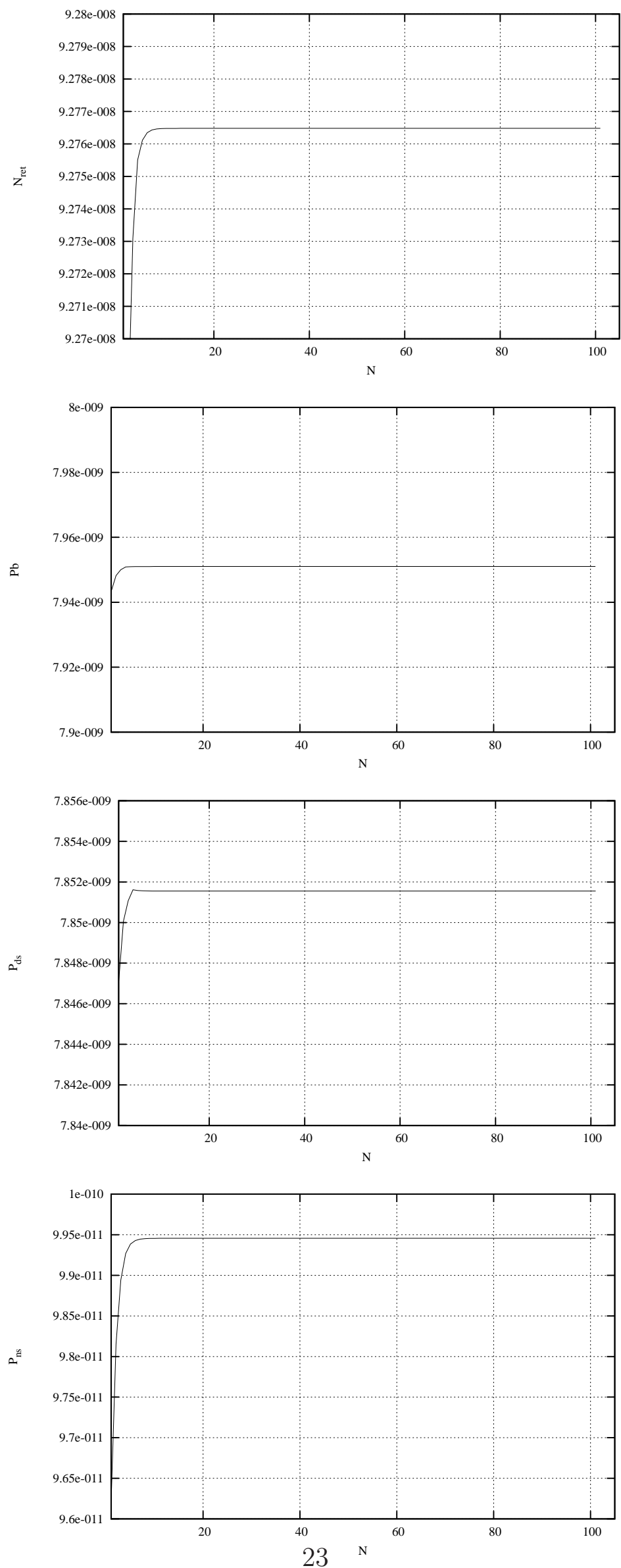

Fig. 3. Performance measures vs $N$ for $c=50, \rho=0.4, \mu=1 / 180, \mu_{r}=0.01$, $P_{i m}=0.2$ 

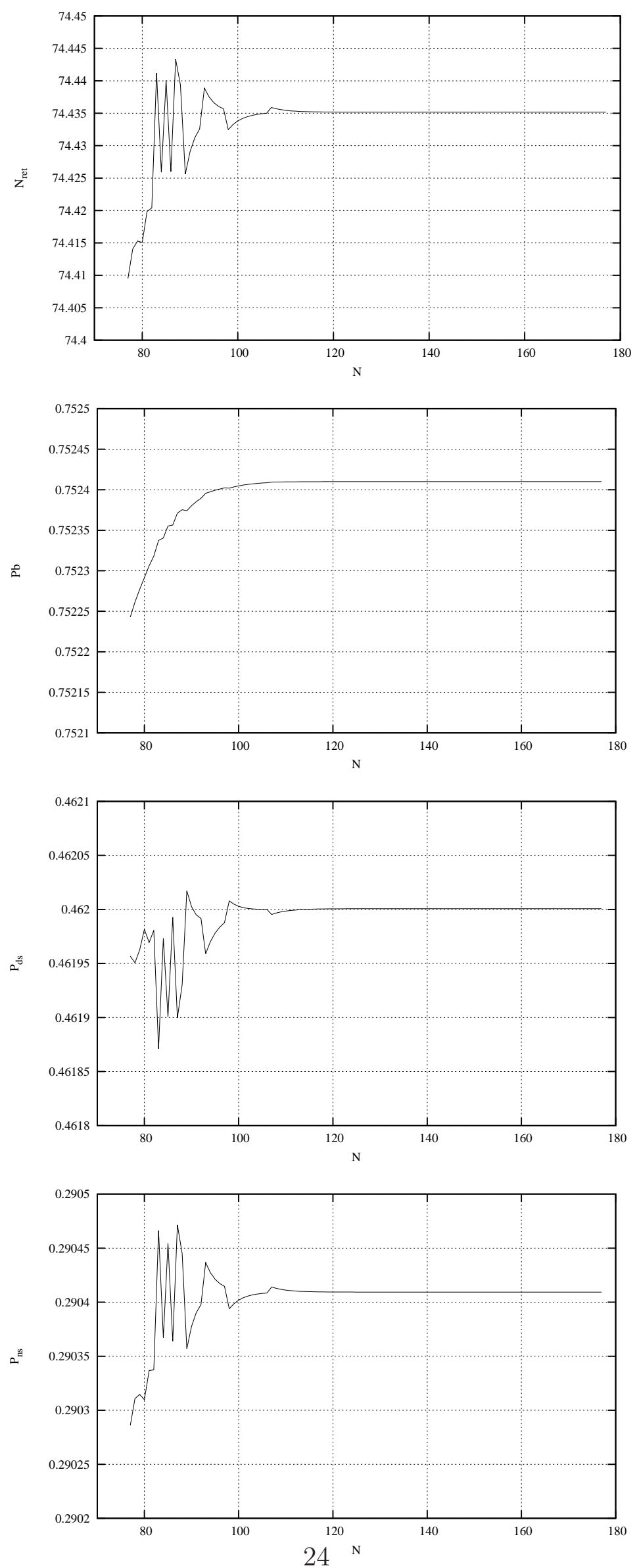

Fig. 4. Performance measures vs $N$ for $c=50, \rho=1.4, \mu=1 / 180, \mu_{r}=0.01$, $P_{i m}=0.2$ 
Table 1

$N$ and computational time of the algorithm for $K=3, L=2, \epsilon_{p}=10^{-5}, \epsilon_{M}=10^{-3}$,

$\underline{\epsilon_{r}=10^{-10}, \rho=\lambda /(\mu c), \mu=1 / 180, \mu_{r}=0.01, P_{i m}=0.2, r_{t h}=0.95}$

\begin{tabular}{|c|c|c|c|c|c|c|c|c|c|c|c|}
\hline \multirow[b]{2}{*}{$\rho$} & & \multicolumn{2}{|c|}{$c=50$} & \multicolumn{2}{|c|}{$c=100$} & \multicolumn{2}{|c|}{$c=200$} & \multicolumn{2}{|c|}{$c=500$} & \multicolumn{2}{|c|}{$c=1000$} \\
\hline & & $N$ & Time (s) & $N$ & Time (s) & $N$ & Time (s) & $N$ & Time (s) & $N$ & Time (s) \\
\hline \multirow[t]{4}{*}{0.4} & $N_{\text {ret }}$ & 17 & 0.889 & 6 & 0.765 & 8 & 1.435 & 39 & 16.318 & 13 & 13.121 \\
\hline & $P_{b}$ & 16 & 0.858 & 4 & 0.468 & 8 & 1.42 & 39 & 16.255 & 13 & 13.275 \\
\hline & $P_{d s}$ & 16 & 0.873 & 5 & 0.609 & 8 & 1.42 & 39 & 16.38 & 13 & 13.166 \\
\hline & $P_{n s}$ & 23 & 1.233 & 23 & 2.574 & 12 & 2.449 & 39 & 16.396 & 13 & 13.12 \\
\hline \multirow[t]{4}{*}{0.8} & $N_{\text {ret }}$ & 38 & 2.511 & 29 & 3.697 & 46 & 9.703 & 35 & 15.646 & 13 & 15.287 \\
\hline & $P_{b}$ & 38 & 2.574 & 32 & 4.57 & 41 & 8.408 & 35 & 15.647 & 12 & 13.744 \\
\hline & $P_{d s}$ & 38 & 2.511 & 32 & 4.446 & 41 & 8.377 & 35 & 15.709 & 11 & 11.762 \\
\hline & $P_{n s}$ & 47 & 3.182 & 32 & 4.617 & 46 & 9.751 & 36 & 17.035 & 40 & 52.355 \\
\hline \multirow[t]{4}{*}{1.0} & $N_{\text {ret }}$ & 44 & 2.855 & 76 & 7.504 & 117 & 14.133 & 206 & 35.491 & 363 & 82.619 \\
\hline & $P_{b}$ & 44 & 2.792 & 76 & 7.769 & 117 & 14.227 & 206 & 35.756 & 363 & 82.509 \\
\hline & $P_{d s}$ & 44 & 2.698 & 76 & 7.737 & 124 & 16.114 & 206 & 35.506 & 363 & 82.914 \\
\hline & $P_{n s}$ & 44 & 2.777 & 79 & 8.129 & 124 & 16.255 & 206 & 36.099 & 363 & 82.477 \\
\hline \multirow[t]{4}{*}{1.4} & $N_{r e t}$ & 102 & 3.042 & 198 & 7.957 & 397 & 26.411 & 858 & 81.417 & 1679 & 264.001 \\
\hline & $P_{b}$ & 100 & 2.621 & 198 & 8.064 & 397 & 26.427 & 858 & 81.589 & 1679 & 266.122 \\
\hline & $P_{d s}$ & 106 & 3.463 & 198 & 8.003 & 397 & 26.318 & 858 & 81.339 & 1679 & 264.781 \\
\hline & $P_{n s}$ & 106 & 3.464 & 202 & 9.375 & 397 & 26.458 & 858 & 81.354 & 1679 & 266.013 \\
\hline
\end{tabular}

computational time complexity is still of $O(c)$ for a specific $\rho$.

Remark. It is worth emphasizing that approaches belonging to the category "Approximations" (Domenech-Benlloch et al. [20]) produced unacceptable errors in most cases. Therefore, we do not focus on the comparison with these algorithms in this paper. The HM2 algorithm overcomes other approaches in the term of the accuracy. Our algorithm has the same accuracy as the HM2. However, it is much faster than the HM2 algorithm. We have shown that the computational time complexity of our algorithm is of $O(c)$. To our best knowledge, we do not know that there is any other algorithm which has the computational time complexity of $O(c)$ for the $\mathrm{M} / \mathrm{M} / \mathrm{c}$ retrial queue with impatient customers and has the same accuracy as of the HM2 algorithm (see Domenech-Benlloch et al. [20] and Do [3] for the overview of the latest algorithms). 


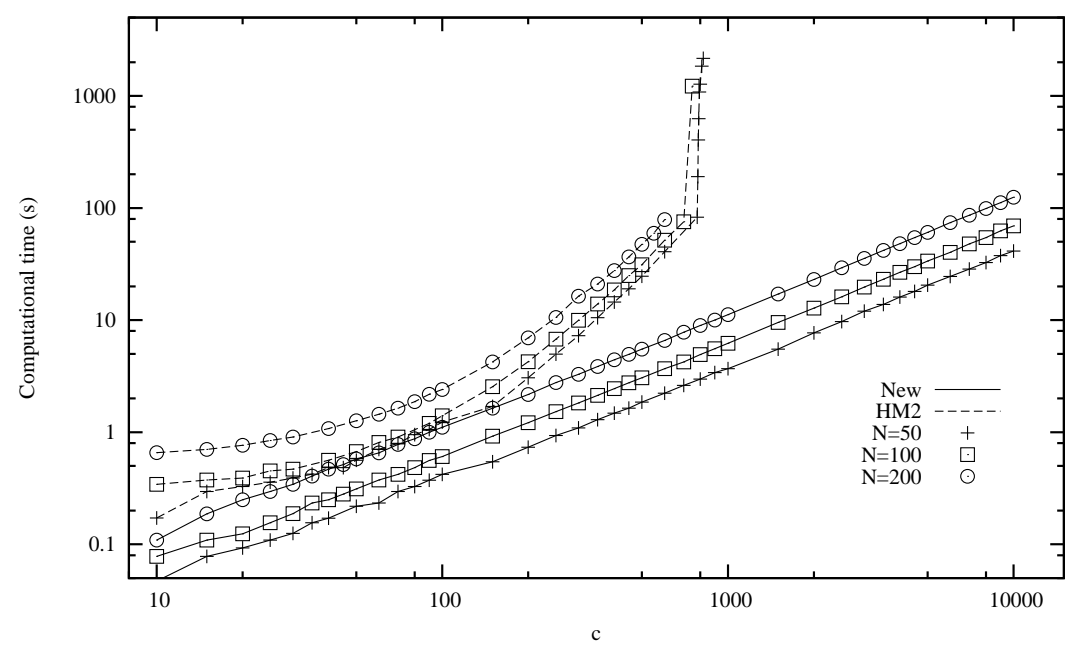

Fig. 5. Computational time vs $c$ for $\mu=1, \mu_{r}=0.5, \rho=\lambda /(\mu c)=0.8, P_{i m}=0.2$, $\epsilon_{M}=10^{-3}$ and $\epsilon_{r}=10^{-10}$

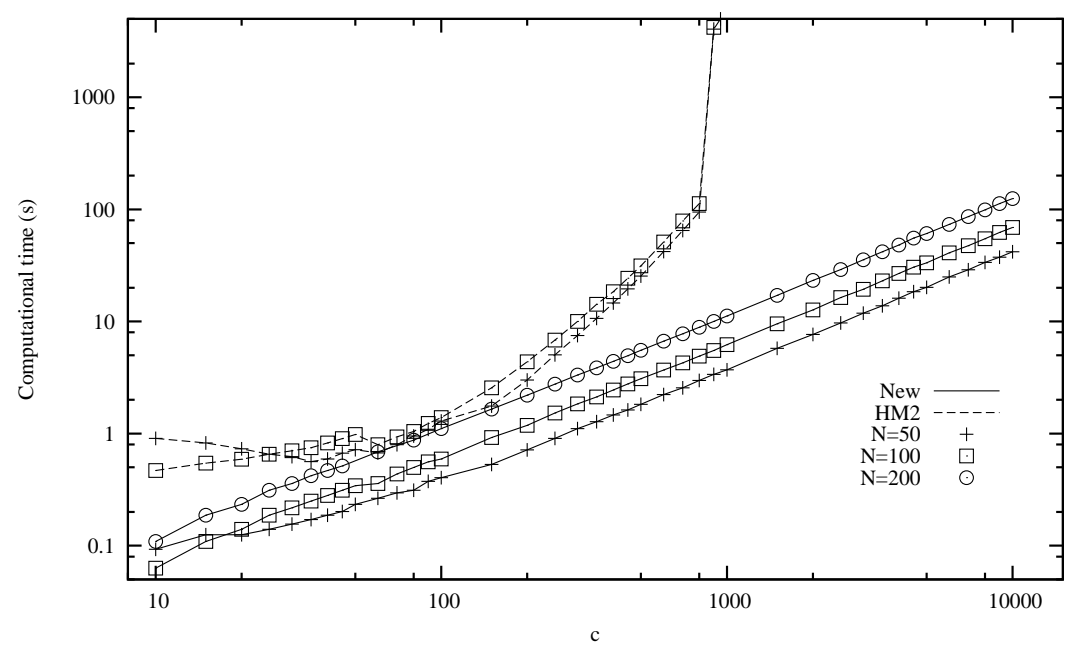

Fig. 6. Computational time vs $c$ for $\mu=1, \mu_{r}=0.05, \rho=\lambda /(\mu c)=0.8, P_{i m}=0.2$, $\epsilon_{M}=10^{-3}$ and $\epsilon_{r}=10^{-10}$

\section{Conclusions}

We have presented our contributions that enable the approximation of the performance of a multiserver retrial queue with impatient customers. We derived exact expressions for the computation of the rate matrix and the conditional mean number of customers. We explore the behavior of performance measures versus $N$, then the estimation of threshold $N$ is derived. We have presented some important properties and provided a proof of concerning the determination of important quantities. Based on the derivations, we have constructed an algorithm to solve the multiserver retrial queue with impatient customers.

In the future, we will investigate the application of the proposed approach for 


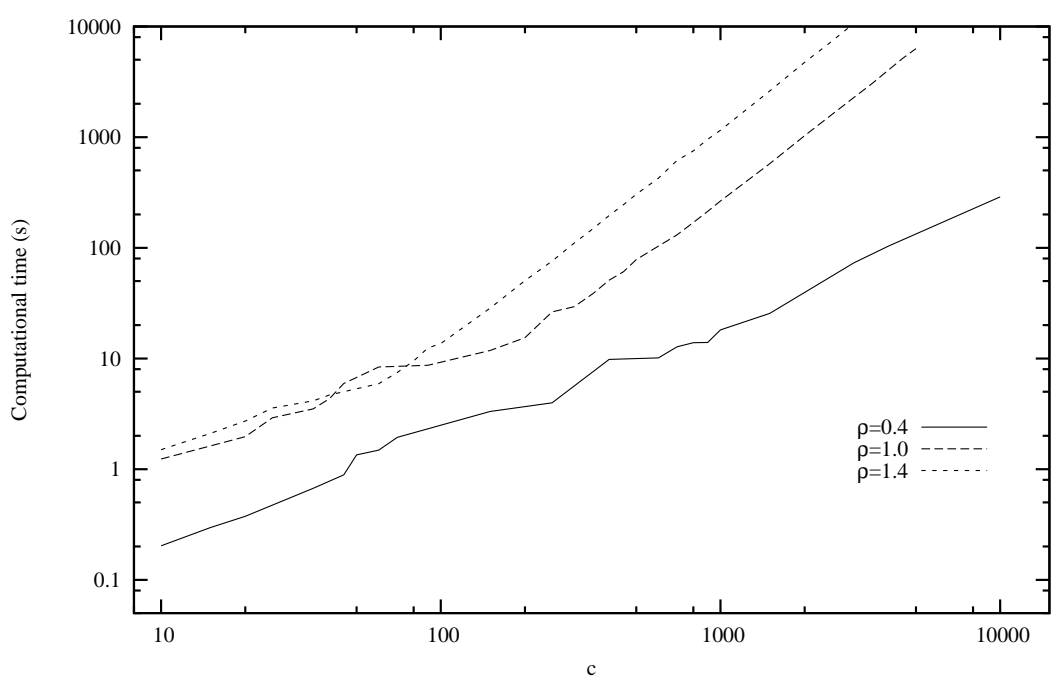

Fig. 7. Computation time vs $c$ and $\rho$ for $K=3, L=2, \mu=1.0, \mu_{r}=0.5, P_{i m}=0.2$, $\epsilon_{M}=10^{-3}, \epsilon_{p}=10^{-5}$ and $\epsilon_{r}=10^{-10}, r_{t h}=0.95$

queueing problems $[3,4]$ in cellular mobile networks.

\section{Acknowledgement}

The publication was supported by the TÁMOP-4.2.2.C-11/1/KONV-20120001 project. The project has been supported by the European Union, cofinanced by the European Social Fund.

The authors are grateful for anonymous reviewers for comments that help to improve the presentation of this paper.

\section{References}

[1] Artalejo, J. R. (1999) Accessible Bibliography on Retrial Queues. Math. Compt. Model, 30, 1-6.

[2] Artalejo, J. R. (2010) Accessible bibliography on retrial queues: Progress in 2000-2009. Mathematical and Computer Modelling, 51, 1071-1081.

[3] Do, T. V. (2010) A new computational algorithm for retrial queues to cellular mobile systems with guard channels. Computers 83 Industrial Engineering, 59, $865-872$.

[4] Do, T. V. (2011) Solution for a retrial queueing problem in cellular networks with the fractional guard channel policy. Mathematical and Computer Modelling, 53(11-12):2058-2065, 2011. 
[5] Do, T. V. and Chakka, R. (2010) An Efficient Method to Compute the Rate Matrix for Retrial Queues with Large Number of Servers, Applied Mathematics Letters, vol. 23, pp. 638-643, 2010.

[6] Falin, G. I. (1990) A Survey of Retrial Queues. Queueing Systems, 7, 127-168.

[7] Falin, G. I. and Templeton, J. G. C. (1997) Retrial Queues. Chapman \& Hall, London.

[8] Artalejo, J. R. and Gómez-Corral, A. (2008) Retrial Queueing Systems. Springer-Verlag, Berlin Heidelberg.

[9] Wüchner, P., Sztrik, J., and de Meer, H. (2009) Finite-source M/M/S retrial queue with search for balking and impatient customers from the orbit. Computer Networks, 53, 1264-1273.

[10] Wang, J., Cao, J., and Li, Q. (2001) Reliability analysis of the retrial queue with server breakdowns and repairs. Queueing Syst., 38, 363-380.

[11] Artalejo, J. and Pozo, M. (2002) Numerical calculation of the stationary distribution of the main multiserver retrial queue. Annals of Operations Research, 116, 41-56. 10.1023/A:1021359709489.

[12] Yang, T. and Templeton, J. G. C. (1982) A Survey on Retrial Queues. Queueing Systems, 2, 201-233.

[13] Falin, G. I. (1984) On Sufficient Conditions for Ergodicity of Multichannel Queueing Systems with Repeated Calls. Adv. Appl. Prob., 16, 447-448.

[14] Neuts, M. F. and Rao, B. M. (1990) Numerical investigation of a multiserver retrial model. Queueing Syst., 7, 169-189.

[15] Neuts, M. F. (1981) Matrix Geometric Soluctions in Stochastic Model. Johns Hopkins University Press, Baltimore.

[16] Latouche, G. and Ramaswami, V. (1999) Introduction to Matrix Analytic Methods in Stochastic Modeling. ASA-SIAM Series on Statistics and Applied Probability.

[17] Mitrani, I. and Chakka, R. (1995) Spectral expansion solution for a class of Markov models: Application and comparison with the matrix-geometric method. Performance Evaluation, 23, 241-260.

[18] Bini, D. A., Latouche, G., and Meini, B. (2005) Numerical Methods for Structured Markov Chains (Numerical Mathematics and Scientific Computation). Oxford University Press, Inc., New York, NY, USA.

[19] Naoumov, V., Krieger, U. R., and Warner, D. (1997) Analysis of a MultiServer Delay-Loss System With a General Markovian Arrival Process. In Chakravarthy, S. R. and Alfa, A. S. (eds.), Matrix-analytic methods in stochastic models, September, Lecture Notes in Pure and Applied Mathematics, 183, pp. 43-66. Marcel Dekker. 
[20] Domenech-Benlloch, M. J., Gimenez-Guzman, J. M., Pla, V., Martinez-Bauset, J., and Casares-Giner, V. (2008) Generalized truncated methods for an efficient solution of retrial systems. Mathematical Problems in Engineering, (2008), doi:10.1155/2008/183089, 2008.

[21] Kleinrock, L. (1975) Queueing Systems: Vol I. Wiley.

[22] Press, W. H., Teukolsky, S. A., Vetterling, W. T., and Flannery, B. P. (2007) Numerical Recipes 3rd Edition: The Art of Scientific Computing. Cambridge University Press, New York, NY, USA.

[23] Miller, K. S. (1981) On the inverse of the sum of matrices. Mathematics Magazine, 54, 67-72.

[24] Conte, S. and deBoor, C. (1972) Elementary Numerical Analysis. McGraw-Hill, New York. 
An Enhanced Algorithm to Solve Multiserver Retrial Queueing Systems with Impatient Customers e-companion 

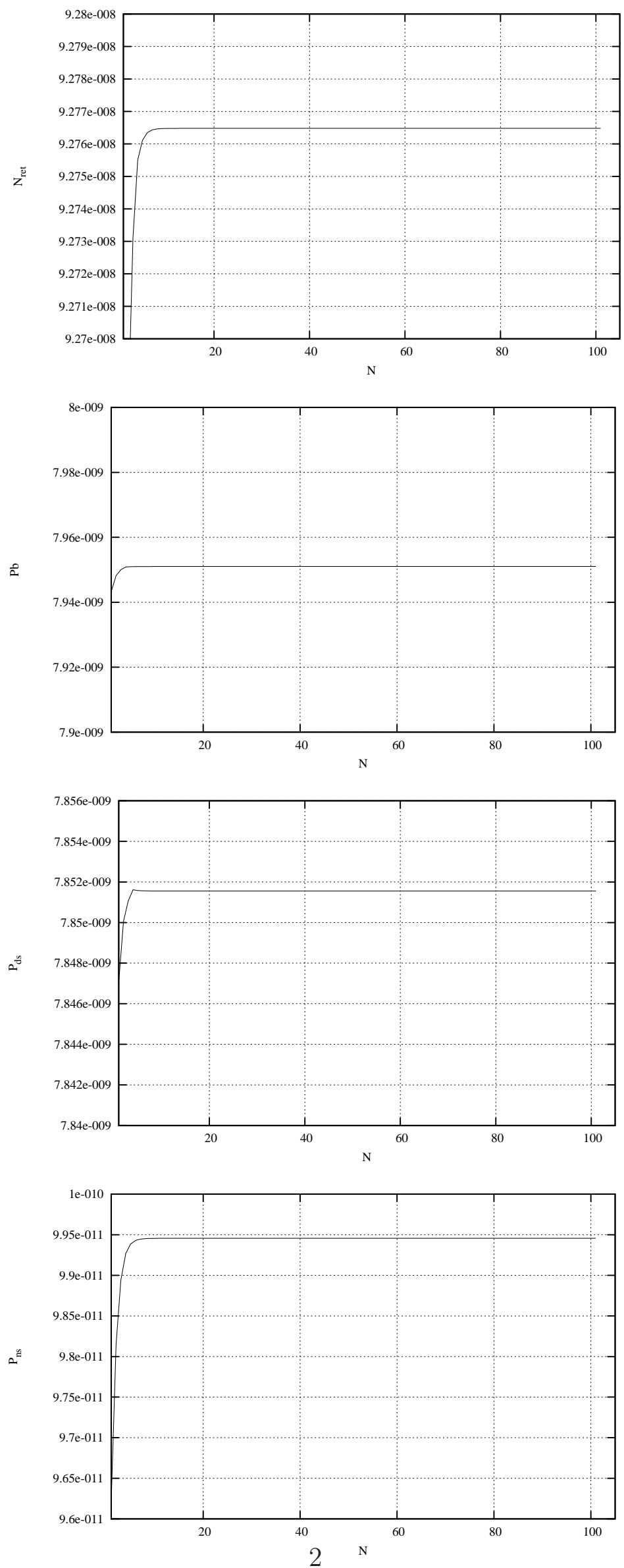

Fig. 1. Performance measures vs $N$ for $c=50, \rho=0.4, \mu=1 / 180, \mu_{r}=0.01$, $P_{i m}=0.2$ 

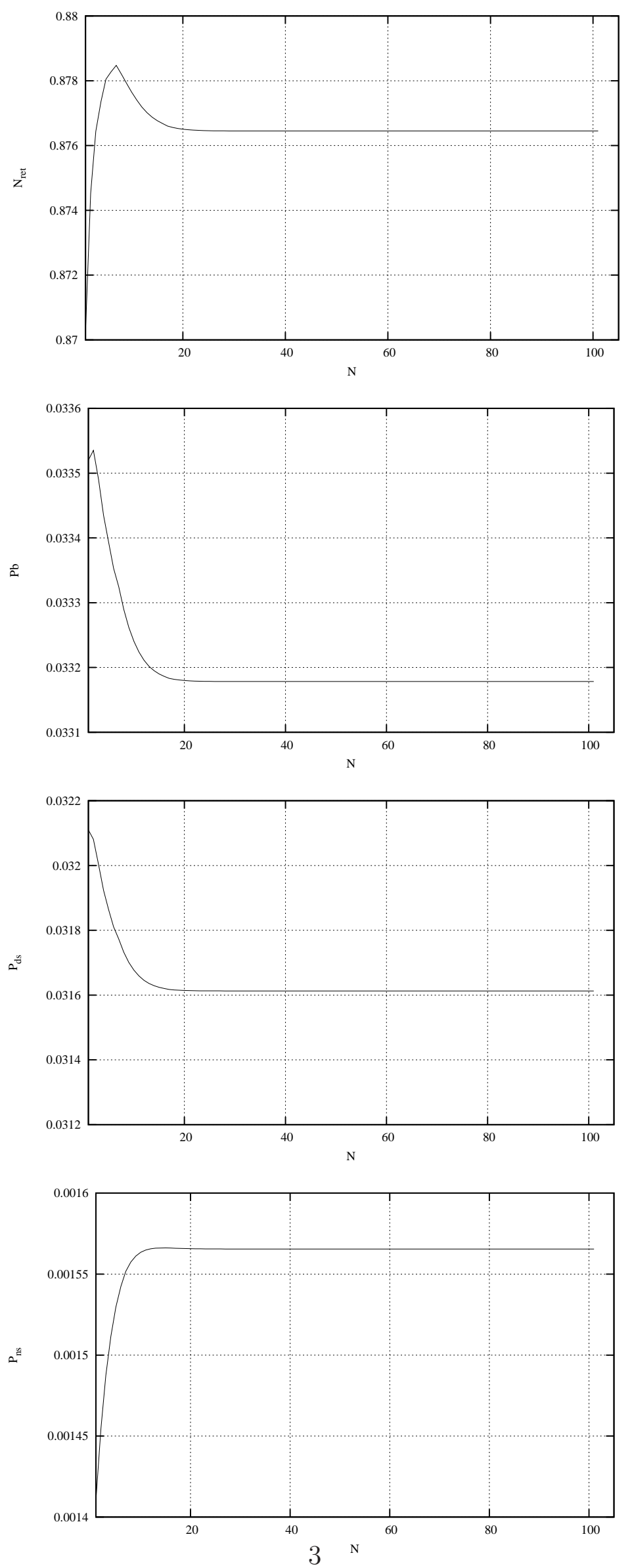

Fig. 2. Performance measures vs $N$ for $c=50, \rho=0.8, \mu=1 / 180, \mu_{r}=0.01$, $P_{i m}=0.2$ 

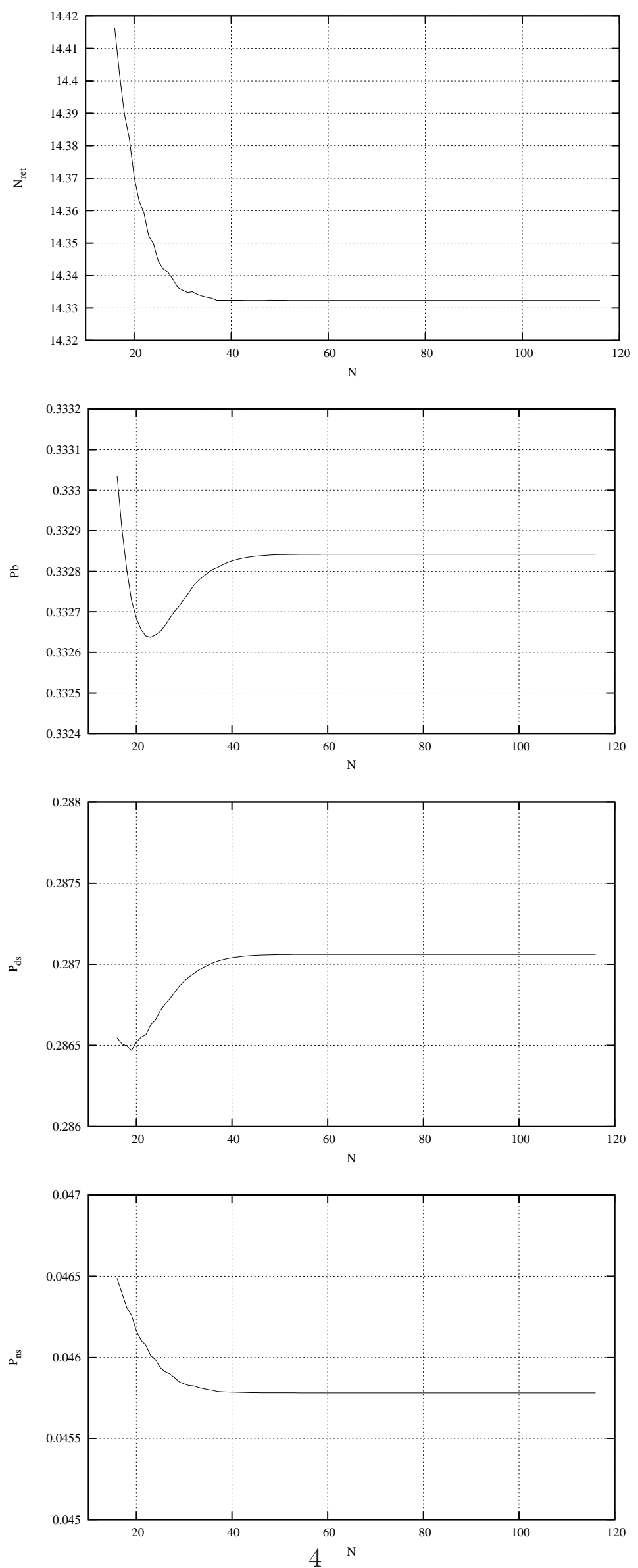

Fig. 3. Performance measures vs $N$ for $c=50, \rho=1.0, \mu=1 / 180, \mu_{r}=0.01$, $P_{i m}=0.2$ 

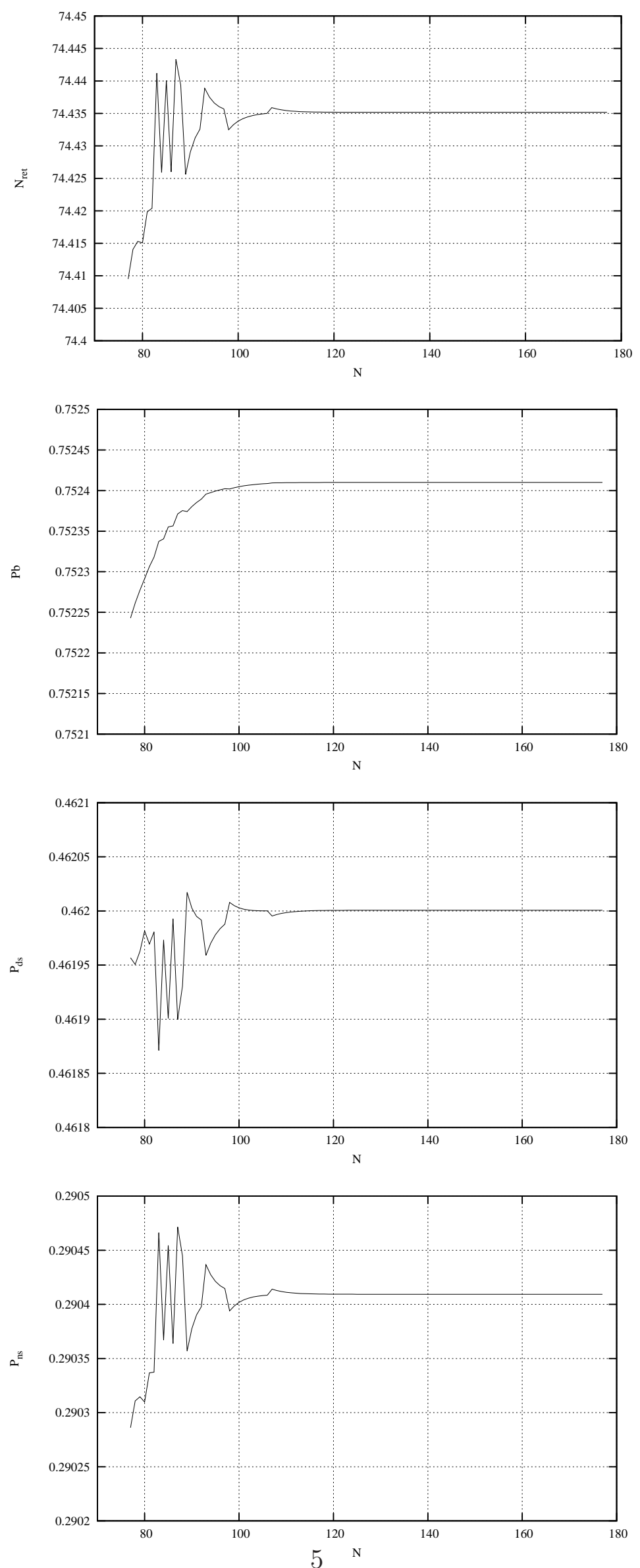

Fig. 4. Performance measures vs $N$ for $c=50, \rho=1.4, \mu=1 / 180, \mu_{r}=0.01$, $P_{i m}=0.2$ 

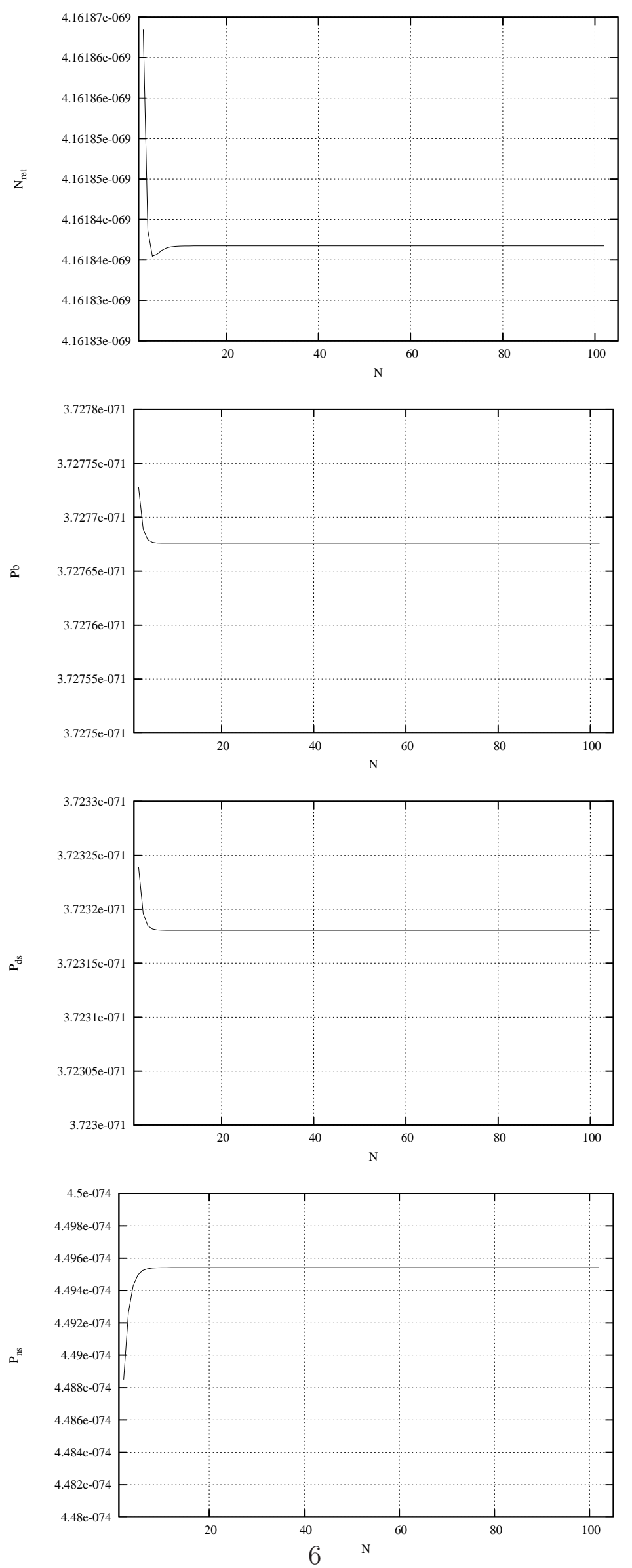

Fig. 5. Performance measures vs $N$ for $c=500, \rho=0.4, \mu=1 / 180, \mu_{r}=0.01$, $P_{i m}=0.2$ 

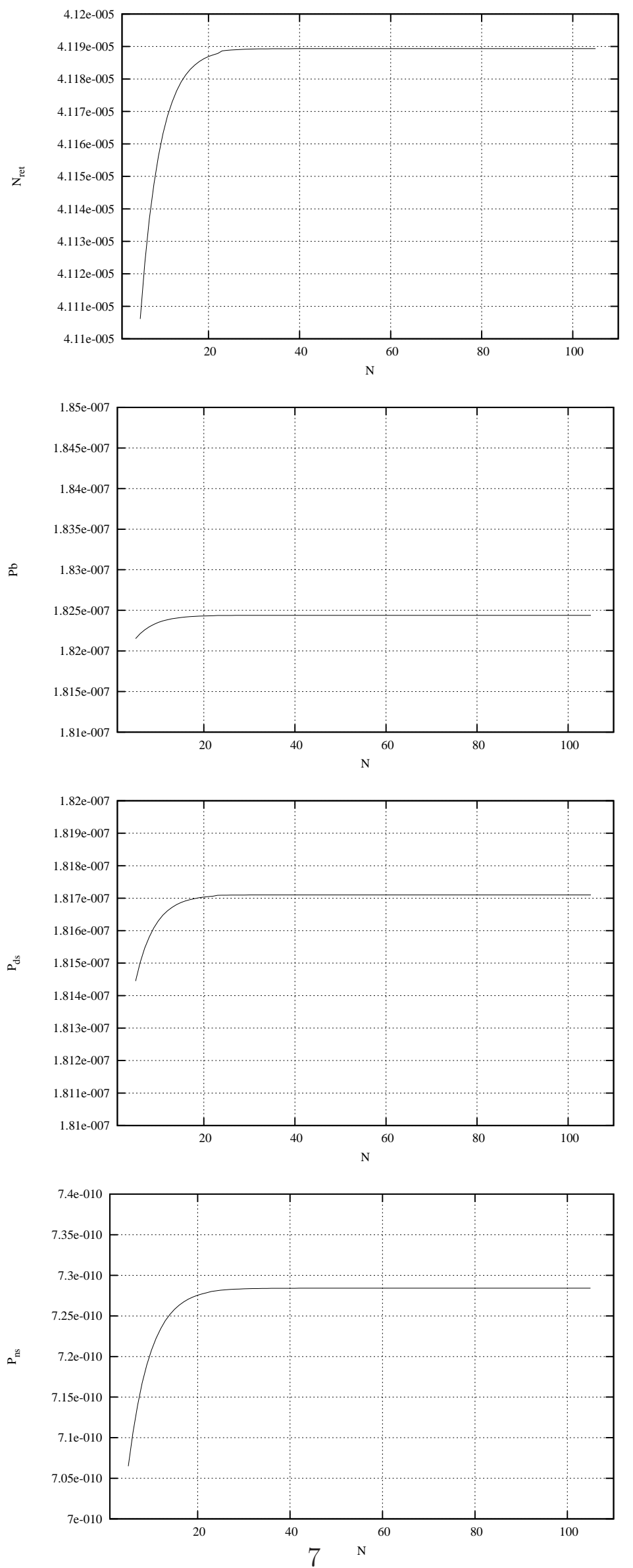

Fig. 6. Performance measures vs $N$ for $c=500, \rho=0.8, \mu=1 / 180, \mu_{r}=0.01$, $P_{i m}=0.2$ 

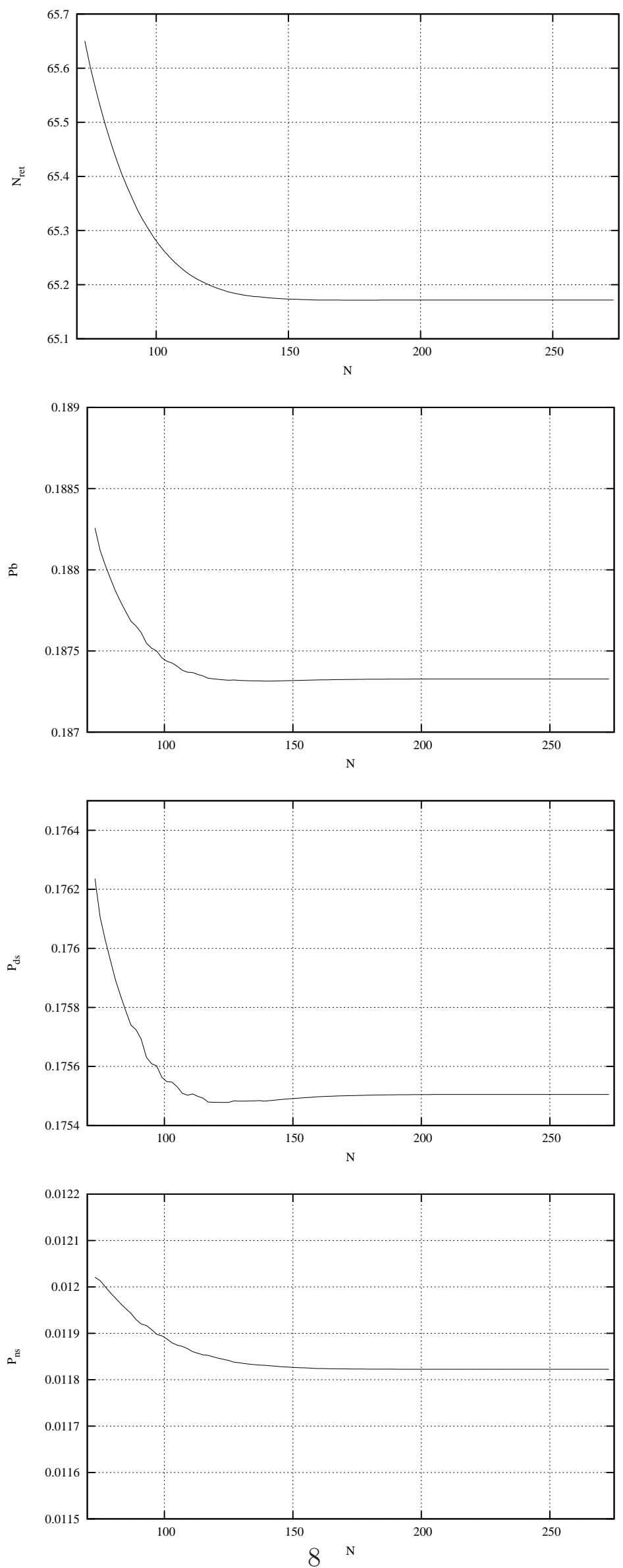

Fig. 7. Performance measures vs $N$ for $c=500, \rho=1.0, \mu=1 / 180, \mu_{r}=0.01$, $P_{i m}=0.2$ 

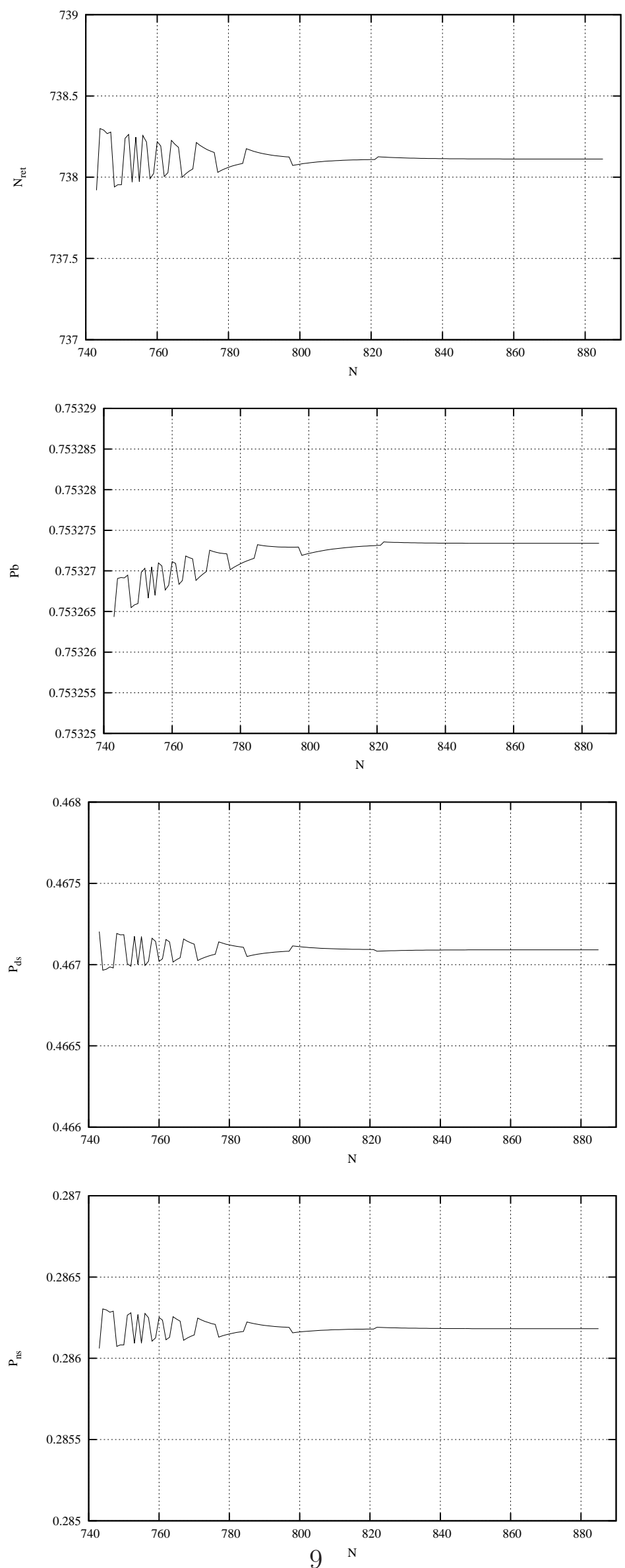

Fig. 8. Performance measures vs $N$ for $c=500, \rho=1.4, \mu=1 / 180, \mu_{r}=0.01$, $P_{i m}=0.2$ 
Table 1

$N$ and the computational time of the proposed algorithm for $K=3, L=2 \epsilon_{p}=$ $10^{-5}, \epsilon_{M}=10^{-3}, \epsilon_{r}=10^{-10}, \rho=\lambda /(\mu c), \mu=1 / 180, \mu_{r}=0.01, P_{i m}=0.2$, $\underline{r_{t h}}=0.95$

\begin{tabular}{|c|c|c|c|c|c|c|c|c|c|c|c|}
\hline \multirow[b]{2}{*}{$\rho$} & & \multicolumn{2}{|c|}{$c=50$} & \multicolumn{2}{|c|}{$c=100$} & \multicolumn{2}{|c|}{$c=200$} & \multicolumn{2}{|c|}{$c=500$} & \multicolumn{2}{|c|}{$c=1000$} \\
\hline & & $N$ & Time (s) & $N$ & Time (s) & $N$ & Time (s) & $N$ & Time (s) & $N$ & Time (s) \\
\hline \multirow[t]{4}{*}{0.4} & $N_{\text {ret }}$ & 17 & 0.889 & 6 & 0.765 & 8 & 1.435 & 39 & 16.318 & 13 & 13.121 \\
\hline & $P_{b}$ & 16 & 0.858 & 4 & 0.468 & 8 & 1.42 & 39 & 16.255 & 13 & 13.275 \\
\hline & $P_{d s}$ & 16 & 0.873 & 5 & 0.609 & 8 & 1.42 & 39 & 16.38 & 13 & 13.166 \\
\hline & $P_{n s}$ & 23 & 1.233 & 23 & 2.574 & 12 & 2.449 & 39 & 16.396 & 13 & 13.12 \\
\hline \multirow[t]{4}{*}{0.8} & $N_{\text {ret }}$ & 38 & 2.511 & 29 & 3.697 & 46 & 9.703 & 35 & 15.646 & 13 & 15.287 \\
\hline & $P_{b}$ & 38 & 2.574 & 32 & 4.57 & 41 & 8.408 & 35 & 15.647 & 12 & 13.744 \\
\hline & $P_{d s}$ & 38 & 2.511 & 32 & 4.446 & 41 & 8.377 & 35 & 15.709 & 11 & 11.762 \\
\hline & $P_{n s}$ & 47 & 3.182 & 32 & 4.617 & 46 & 9.751 & 36 & 17.035 & 40 & 52.355 \\
\hline \multirow[t]{4}{*}{1.0} & $N_{r e t}$ & 44 & 2.855 & 76 & 7.504 & 117 & 14.133 & 206 & 35.491 & 363 & 82.619 \\
\hline & $P_{b}$ & 44 & 2.792 & 76 & 7.769 & 117 & 14.227 & 206 & 35.756 & 363 & 82.509 \\
\hline & $P_{d s}$ & 44 & 2.698 & 76 & 7.737 & 124 & 16.114 & 206 & 35.506 & 363 & 82.914 \\
\hline & $P_{n s}$ & 44 & 2.777 & 79 & 8.129 & 124 & 16.255 & 206 & 36.099 & 363 & 82.477 \\
\hline \multirow[t]{4}{*}{1.4} & $N_{r e t}$ & 102 & 3.042 & 198 & 7.957 & 397 & 26.411 & 858 & 81.417 & 1679 & 264.001 \\
\hline & $P_{b}$ & 100 & 2.621 & 198 & 8.064 & 397 & 26.427 & 858 & 81.589 & 1679 & 266.122 \\
\hline & $P_{d s}$ & 106 & 3.463 & 198 & 8.003 & 397 & 26.318 & 858 & 81.339 & 1679 & 264.781 \\
\hline & $P_{n s}$ & 106 & 3.464 & 202 & 9.375 & 397 & 26.458 & 858 & 81.354 & 1679 & 266.013 \\
\hline
\end{tabular}


Table 2

$N$ and the computational time of the proposed algorithm for $K=4, L=2, \epsilon_{p}=$ $10^{-5}, \epsilon_{M}=10^{-3}, \epsilon_{r}=10^{-10}, \rho=\lambda /(\mu c), \mu=1 / 180, \mu_{r}=0.01, P_{i m}=0.2$, $\underline{r_{t h}}=0.95$

\begin{tabular}{|c|c|c|c|c|c|c|c|c|c|c|c|}
\hline \multirow[b]{2}{*}{$\rho$} & & \multicolumn{2}{|c|}{$c=50$} & \multicolumn{2}{|c|}{$c=100$} & \multicolumn{2}{|c|}{$c=200$} & \multicolumn{2}{|c|}{$c=500$} & \multicolumn{2}{|c|}{$c=1000$} \\
\hline & & $N$ & Time (s) & $N$ & Time (s) & $N$ & Time (s) & $N$ & Time (s) & $N$ & Time (s) \\
\hline \multirow[t]{4}{*}{0.4} & $N_{\text {ret }}$ & 23 & 1.061 & 7 & 0.765 & 11 & 1.841 & 43 & 17.504 & 14 & 13.541 \\
\hline & $P_{b}$ & 17 & 0.78 & 5 & 0.484 & 11 & 1.809 & 43 & 17.487 & 14 & 13.588 \\
\hline & $P_{d s}$ & 17 & 0.749 & 5 & 0.499 & 11 & 1.779 & 43 & 17.254 & 14 & 13.462 \\
\hline & $P_{n s}$ & 23 & 1.06 & 36 & 3.213 & 14 & 2.559 & 43 & 17.316 & 14 & 13.447 \\
\hline \multirow[t]{4}{*}{0.8} & $N_{\text {ret }}$ & 47 & 2.808 & 30 & 3.713 & 53 & 10.639 & 36 & 15.694 & 15 & 16.614 \\
\hline & $P_{b}$ & 47 & 2.761 & 34 & 4.352 & 46 & 8.705 & 36 & 15.631 & 12 & 12.465 \\
\hline & $P_{d s}$ & 47 & 2.761 & 34 & 4.337 & 46 & 8.814 & 36 & 15.616 & 12 & 12.387 \\
\hline & $P_{n s}$ & 47 & 2.793 & 34 & 4.305 & 53 & 10.608 & 37 & 16.926 & 43 & 52.057 \\
\hline \multirow[t]{4}{*}{1.0} & $N_{r e t}$ & 52 & 2.933 & 79 & 7.223 & 124 & 14.445 & 225 & 44.959 & 364 & 95.52 \\
\hline & $P_{b}$ & 52 & 2.932 & 79 & 7.332 & 124 & 14.617 & 225 & 44.85 & 364 & 95.02 \\
\hline & $P_{d s}$ & 52 & 2.933 & 79 & 7.363 & 124 & 14.633 & 225 & 44.975 & 364 & 94.584 \\
\hline & $P_{n s}$ & 61 & 3.698 & 83 & 7.971 & 138 & 18.362 & 225 & 44.788 & 364 & 94.833 \\
\hline \multirow[t]{4}{*}{1.4} & $N_{r e t}$ & 106 & 3.042 & 202 & 8.409 & 404 & 28.735 & 863 & 98.421 & 1684 & 332.251 \\
\hline & $P_{b}$ & 102 & 2.62 & 202 & 8.362 & 404 & 28.657 & 863 & 98.515 & 1684 & 331.315 \\
\hline & $P_{d s}$ & 106 & 3.011 & 202 & 8.377 & 404 & 29.343 & 863 & 98.405 & 1684 & 330.16 \\
\hline & $P_{n s}$ & 106 & 3.042 & 202 & 8.362 & 404 & 28.735 & 863 & 98.078 & 1684 & 332.641 \\
\hline
\end{tabular}


Table 3

$N$ and the computational time of the proposed algorithm for $K=5, L=2, \epsilon_{p}=$ $10^{-5}, \epsilon_{M}=10^{-3}, \epsilon_{r}=10^{-10}, \rho=\lambda /(\mu c), \mu=1 / 180, \mu_{r}=0.01, P_{i m}=0.2$, $\underline{r_{t h}}=0.95$

\begin{tabular}{|c|c|c|c|c|c|c|c|c|c|c|c|}
\hline \multirow[b]{2}{*}{$\rho$} & & \multicolumn{2}{|c|}{$c=50$} & \multicolumn{2}{|c|}{$c=100$} & \multicolumn{2}{|c|}{$c=200$} & \multicolumn{2}{|c|}{$c=500$} & \multicolumn{2}{|c|}{$c=1000$} \\
\hline & & $N$ & Time (s) & $N$ & Time (s) & $N$ & Time (s) & $N$ & Time (s) & $N$ & Time (s) \\
\hline \multirow[t]{4}{*}{0.4} & $N_{\text {ret }}$ & 29 & 1.389 & 14 & 1.295 & 12 & 2.122 & 55 & 23.447 & 15 & 15.351 \\
\hline & $P_{b}$ & 23 & 1.045 & 6 & 0.655 & 12 & 2.122 & 55 & 23.447 & 15 & 15.21 \\
\hline & $P_{d s}$ & 23 & 1.061 & 6 & 0.624 & 12 & 2.138 & 55 & 23.416 & 15 & 15.288 \\
\hline & $P_{n s}$ & 29 & 1.341 & 37 & 3.447 & 18 & 3.245 & 55 & 23.588 & 15 & 15.288 \\
\hline \multirow[t]{4}{*}{0.8} & $N_{\text {ret }}$ & 49 & 2.932 & 32 & 3.993 & 56 & 11.715 & 37 & 17.316 & 16 & 18.642 \\
\hline & $P_{b}$ & 49 & 2.98 & 45 & 5.553 & 53 & 10.671 & 37 & 16.973 & 13 & 14.055 \\
\hline & $P_{d s}$ & 49 & 2.979 & 45 & 5.491 & 53 & 10.686 & 37 & 17.051 & 13 & 14.04 \\
\hline & $P_{n s}$ & 49 & 2.949 & 45 & 5.569 & 56 & 12.137 & 40 & 19.126 & 49 & 59.717 \\
\hline \multirow[t]{4}{*}{1.0} & $N_{\text {ret }}$ & 54 & 3.198 & 83 & 7.94 & 138 & 18.346 & 229 & 52.573 & 386 & 134.051 \\
\hline & $P_{b}$ & 54 & 3.198 & 83 & 8.003 & 138 & 18.58 & 229 & 52.65 & 386 & 132.102 \\
\hline & $P_{d s}$ & 54 & 3.104 & 83 & 8.018 & 138 & 18.626 & 229 & 52.276 & 386 & 133.583 \\
\hline & $P_{n s}$ & 54 & 3.182 & 83 & 8.05 & 140 & 20.03 & 229 & 52.261 & 386 & 133.162 \\
\hline \multirow[t]{4}{*}{1.4} & $N_{\text {ret }}$ & 111 & 3.573 & 204 & 9.547 & 407 & 32.947 & 872 & 123.319 & 1688 & 406.523 \\
\hline & $P_{b}$ & 106 & 3.01 & 204 & 9.454 & 407 & 32.76 & 872 & 123.287 & 1688 & 409.581 \\
\hline & $P_{d s}$ & 111 & 3.51 & 204 & 9.531 & 407 & 33.166 & 872 & 121.665 & 1688 & 411.032 \\
\hline & $P_{n s}$ & 111 & 3.573 & 204 & 9.563 & 407 & 32.838 & 872 & 122.321 & 1688 & 408.005 \\
\hline
\end{tabular}

